This is a post-peer-review, pre-copyedit version of an article published in Space Science Reviews. The final authenticated version is available online at: https://doi.org/10.1007/s11214-019-0585-y

This version is subjected to Springer Nature terms for reuse that can be found at: $h$ ttps://www.springer.com/gp/openaccess/authors-rights/aam-terms-v1 


\title{
Shocks and non-thermal particles in clusters of galaxies
}

\author{
A.M.Bykov, F.Vazza, J.A.Kropotina, \\ K.P.Levenfish, F.B.S.Paerels
}

Received: date / Accepted: date

\begin{abstract}
Galaxy clusters grow by gas accretion, mostly from mergers of substructures, which release powerful shock waves into cosmic plasmas and convert a fraction of kinetic energy into thermal energy, amplification of magnetic fields and into the acceleration of energetic particles. The modeling of the radio signature of cosmic shocks, combined with the lack of detected $\gamma$-rays from cosmic ray (CR) protons, poses challenges to our understanding of how cosmic rays get accelerated and stored in the intracluster medium. Here we review the injection of CRs by cosmic shocks of different strengths, combining the detailed "microscopic" view of collisionless processes governing the creation of non-thermal distributions of electrons and protons in cluster shocks (based on analytic theory and particle-in-cell simulations), with the "macroscopic" view of the large-scale distribution of cosmic rays, suggested by modern cosmological simulations. Time dependent non-linear kinetic models of particle acceleration by multiple internal shocks with large scale compressible motions of plasma with soft CR spectra containing a noticable energy density in the superthermal protons of energies below a few $\mathrm{GeV}$ which is difficult to constrain by Fermi
\end{abstract}

A.M. Bykov

Ioffe Institute, 194021, St. Petersburg, Russia;

E-mail: byk@astro.ioffe.ru

F. Vazza

Dipartimento di Fisica e Astronomia, Universit di Bologna, Via Gobetti 93/2, Italy;

Hamburger Sternwarte, Universität Hamburg, Gojenbergsweg 116, 40129 Hamburg, Germany

J.A. Kropotina

Ioffe Institute, 194021, St. Petersburg, Russia

K.P. Levenfish

Ioffe Institute, 194021, St. Petersburg, Russia

F.B.S. Paerels

Columbia Astrophysics Laboratory Columbia University 550 West 120th Street, New York NY 10027

USA

E-mail: frits@astro.columbia.edu 
observations are discussed. We consider the effect of plasma composition on CR injection and super-thermal particle population in the hot intracluster matter which can be constrained by fine high resolution X-ray spectroscopy of Fe ions.

Keywords Clusters of galaxies $\cdot$ Shocks $\cdot$ Cosmic rays

\section{Plasma shocks in structure formation}

Accurate estimations of the energy content in the non-thermal components (cosmic rays and magnetic fields, beside turbulent gas motions) in clusters of galaxies are needed to allow for "precision cosmology" using galaxy clusters (see Pratt et al this topical collection). Direct evidence of the presence of the non-thermal components came from the radio observations of the extended to Mpc-size synchrotron emission in clusters of galaxies (see e.g. Miley, 1980; Ferrari et al, 2008, van Weeren et al, 2010; Feretti et al, 2012; Brunetti and Jones, 2014), while gamma-ray observations have so far only produced upper limits on the level of diffuse hadronic emission from the intracluster medium (ICM). Hence non detections of hadronic $\gamma$-rays from cosmic rays in clusters have in principle the potential not only to limit the total budget of cosmic rays in clusters (Reimer et al, 2003, Brunetti et al, 2007a; Ackermann et al, 2010, The Fermi-LAT Collaboration et al, 2013; Ackermann et al, 2014, Griffin et al, 2014, Ackermann et al, 2016; Prokhorov and Churazov, 2014; Zandanel and Ando, 2014, Zandanel et al, 2015; Brunetti, 2017) ${ }^{1}$, but also to limit the acceleration efficiency by merger shocks (Vazza and Brüggen, 2014, Vazza et al, 2015). These can constrain the magnetic fields in clusters as well as the parameter space of the models of (re)acceleration of relativistic protons and their secondaries by turbulence in clusters (see e.g. Brunetti et al 2017).

The growth of cosmic structures cannot happen without the formation of shock waves, at whose surface kinetic energy gets dissipated into gas thermalization (e.g. Sunyaev and Zeldovich, 1972, Markevitch and Vikhlinin, 2007, Bykov et al, 2008a Kushnir and Waxman, 2009, Brüggen et al, 2012, Cavaliere and Lapi, 2013). Around galaxy clusters, strong $\mathscr{M} \geq 10-10^{2}$ accretion shocks should exist in a quasi-stationary way and mark the transition from smooth infalling matter to halos undergoing their virialisation process, as illustrated in the top panels of Fig 1, (Miniati et al, 2000; Ryu et al, 2003b, Pfrommer et al, 2006, Vazza et al, 2009, Planelles and Quilis, 2013, Schaal et al, 2016, Martin-Alvarez et al, 2017). The statistics of shocks in the Universe is predicted to be dominated by structure formation shocks, with only a minor contribution from non-gravitational processes, as shown in the bottom left panel of Fig.1 (e.g. Kang et al, 2012a, Vazza et al, 2014, Schaal et al, 2016).

Beside minor differences related to the different adopted hydrodynamical solvers and shock finders, cosmological simulations generally agree that bulk of kinetic energy in the cosmic volume gets dissipated by $2 \leq \mathscr{M} \leq 4$ shocks within large-scale structures (see bottom right panel of Fig 1 and Vazza et al 2011, for a comparison of

\footnotetext{
1 It shall be noted that these constraints are complementary to the ones that can be derived from radio analysis (Dolag and Enßlin 2000, Pfrommer and Enßlin 2004, Brunetti et al. 2007b. Donnert et al| 2010 \begin{tabular}{|l||l|l|l|}
\hline Brown et al 2011, Brunetti and Lazarian 2011a Brunetti et al 2012), in which however the limits are
\end{tabular} intertwined with the uncertainties on the intracluster magnetic fields.
} 

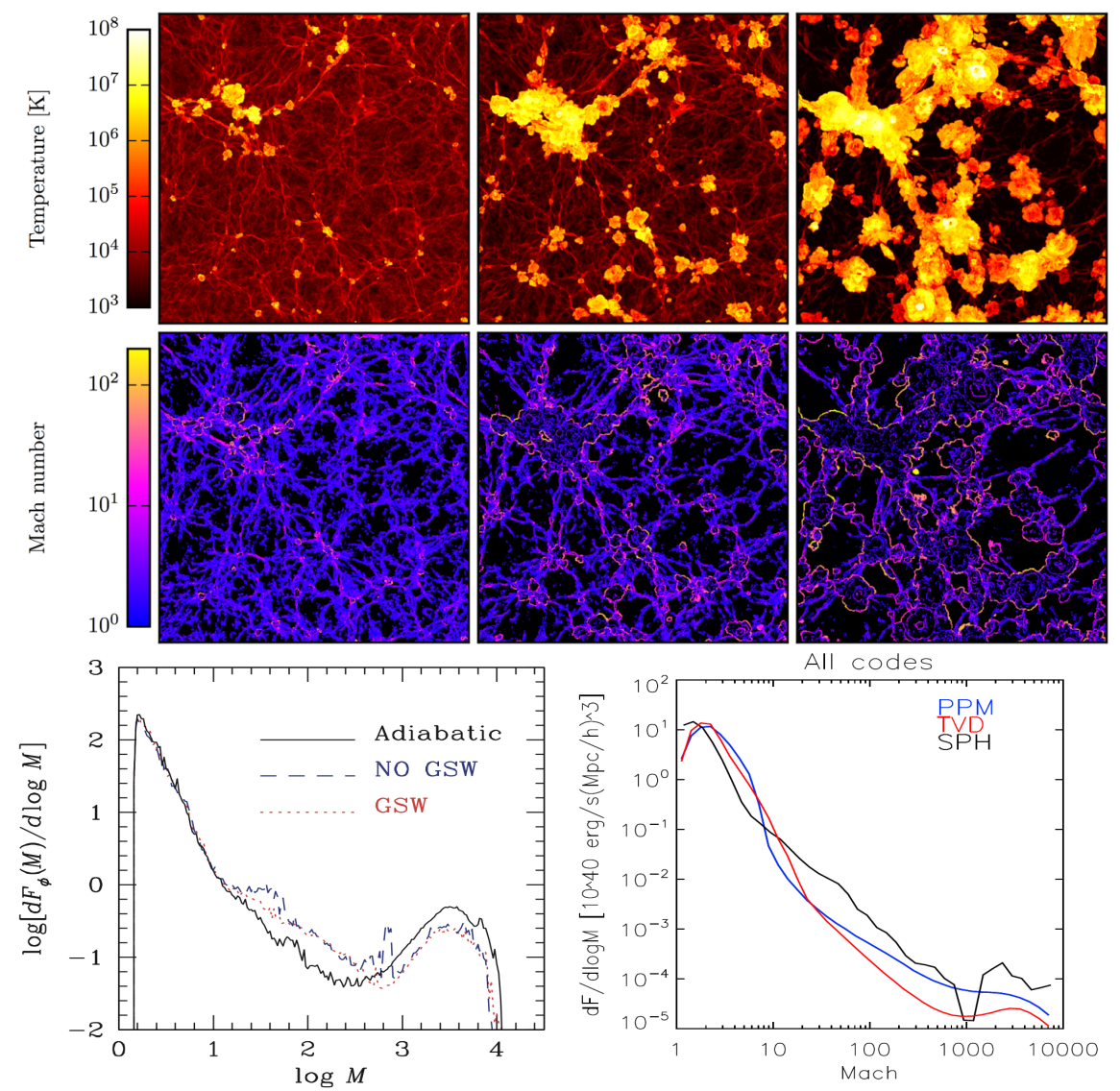

Fig. 1 Spatial distribution and properties of shock waves on cosmological scales based on simulations. Top panels: evolution of gas temperature and shock Mach number for a thin slice crossing the Illustris-1 box at $\mathrm{z}=2, \mathrm{z}=1$ and $\mathrm{z}=0$, from Schaal et al (2016). Bottom left panel: inverse of the mean comoving distance between shock surfaces at $\mathrm{z}=0$ as a function of Mach number in a cosmic volume simulated by Kang et al (2007), comparing an "adiabatic" (i.e. non radiative model) with a radiative model with (GSW) or without (NO GSW) feedback from galactic star wind. Bottom right: distribution of dissipated kinetic energy flux as a function of shocks Mach number identified in three resimulations of the same $(100 \mathrm{Mpc} / \mathrm{h})^{3}$ volume at $\mathrm{z}=0$, employing three different numerical methods: ENZO Eulerian simulations with the Piecewise Parabolic Method (PPM), Eulerian simulations with the Total variation diminishing (TVD) method (Ryu et al 2003b), and Smoothed Particle Hydrodynamics (SPH) simulations with GADGET (Pfrommer et al 2006), adapted from Vazza et al (2011)

cosmological codes). This range of shock strength is intriguingly close to the typically one inferred for shocks associated with observed radio relics (see Van Weeren et al., this topical collection). Such shocks are often associated with major mergers, and these events are expected to maximally enrich the intracluster medium with cosmic rays. The typical regime of merger shocks is also the one at which making predictions of shock acceleration gets more difficult, owing to a number of physical and numerical uncertainties (see Sections below), hence the comparisons with real $\gamma$-ray observations (or upper limits) are particularly important to test "micro"-details 
of shock acceleration models.

We notice that galaxy clusters represent an important testbed for any model attempting to describe the acceleration of cosmic ray protons by structure formation shocks, in a plasma regime complementary to supernova remnants and solar wind shocks. This stems from the fact that, once accelerated, relativistic cosmic ray protons should be long-lived as they are subject to negligible energy losses, and must remain confined inside clusters for cosmological timescales (Völk et al, 1996, Berezinsky et al, 1997, Völk and Atoyan, 1999). $\gamma$-ray observations (or lack thereof) of the diffuse hadronic emission from the intracluster medium have in principle the potential of testing theoretical scenarios of shock acceleration, because they can constrain the time-integrated budget of cosmic rays, injected over the entire lifetime of galaxy clusters.

In addition to shocks, acceleration and re-acceleration of both electrons and nuclei by the compressible large scale plasma motions (where the energy containing scale of the turbulence is longer than the CR mean free paths) were both proposed as an efficient Fermi type CR acceleration mechanism (see Bykov and Toptygin, 1990. 1993; Bykov et al, 2000, Brunetti and Lazarian, 2007, 2011b, and the references therein). Such a large scale turbulence can be induced by the merging and accretion of the cluster sub-structures.

The key problem of the cosmic ray modeling in clusters of galaxies is the transport of CRs, which is governed by the magnetic fluctuations of very different scales which span about ten decades. Strong accretion shocks can convert about $10 \%$ of their kinetic power to amplify magnetic fields and to built the extended spectra of magnetic fluctuations (see e.g. Bykov et al, 2014) as it is likely the case in young supernova remnants. Precise microscopic modeling of the magnetic fluctuation spectra which are produced by both the power of the cluster formation and the different plasma instabilities during the cluster relaxation is non feasible now because it involves the very broad dynamical range of the fluctuations. Physics of the MHD waves in the hot cluster plasmas with high $\beta=8 \pi n k_{B}\left(T_{e}+T_{i}\right) / B^{2}$ (where $n$ is particle number density, electron and ion temperatures are $T_{e}$ and $T_{i}$ and $B$ is magnetic field) is a subject of the current interest (Wiener et al, 2018; Xu and Lazarian, 2018).

We discuss the damping rates of the plasma long wavelength fluctuations to estimate the life time of the turbulence in section 2 Then in sections $3-4$ we consider CR injection and acceleration. In section 5 we briefly discuss how cosmological simulations of evolving galaxy clusters have assisted the theoretical interpretation of $\gamma$-ray limits from galaxy clusters.

\section{Plasma physics of the long-wavelength motions in clusters of galaxies}

The Coulomb mean free part of a thermal ion in a plasma of number density $n_{-3}$ (measured in the units of $10^{-3} \mathrm{~cm}^{-3}$ and temperature $T_{8}$ (measured in $10^{8} \mathrm{~K}$ ) can be estimated as $\lambda_{C} \approx 17 T_{8}^{2} / n_{-3} \mathrm{kpc}$. Note that at the redshifts $z<0.05$, the superb Chandra's angular resolution corresponds to linear sizes of less than a kiloparsec. 
Therefore it is possible to study in X-rays different collisionless structures in the intercluster medium (see e.g. Markevitch and Vikhlinin, 2007; Walker et al, 2018).

Compressible magnetosonic wave of a frequency $\omega$ propagating in high $\beta$ collisionless plasma is a subject of a strong Landau damping. In an isothermal maxwellian plasma with $T_{e}=T_{i}$ a fast magnetosonic wave of a phase velocity $v_{\mathrm{ph}}$ propagating at an angle $\theta$ to the mean homogeneous magnetic field of a magnitude $B_{0}$ would have a linear damping rate $\gamma$ :

$$
\frac{\gamma_{\mathrm{ms}}}{\omega}=\sqrt{\frac{\pi}{4}} \frac{v_{\mathrm{Ti}}}{v_{\mathrm{ph}}} \frac{\sin ^{2} \theta}{|\cos \theta|}\left[\sqrt{\frac{m_{e}}{m_{p}}}+5 \exp \left(-\frac{v_{\mathrm{Ti}}^{2}}{v_{\mathrm{ph}}^{2} \cos ^{2} \theta}\right)\right] .
$$

Therefore in high $\beta$ Maxwellian plasma where the ion thermal velocity $v_{\mathrm{Ti}}>>$ $v_{\mathrm{ph}}$ only nearly parallel propagating magnetosonic waves of $\theta<<1$ can survive according to the linear approximation given by Eq. 1. However, the nonlinear effects of particle trapping, particle energy change due to scattering and trapping can result in a deformation of the initially maxwellian distribution function around the Landau resonant momenta (O'Neil, 1965, Toptygin, 1985; Matsumoto, 1977; Mouhot and Villani, 2010, Artemyev et al, 2018). Some fraction of the particles can be captured between crests of the wave and these particles do not change the energy of the wave. Only uncaptured (transit) particles at the Landau resonant region efficiently take off the energy of the wave. In this regime the wave damping is determined by the collision rate $v_{\text {coll }}$ which regulates the exchange between the captured and uncaptured particle populations (Toptygin, 1985). The rate $v_{\text {coll }}$ can be either the Coulomb collision rate (which is rather low in the ICM) or the particle scattering rate by collisionless magnetic fuctuations. The linear regime of strong Landau damping will occure if the effective collisions are capable of restoring the Maxwellian particle distribution over the time period of the particle oscillation. Otherwise, the wave damping may be strongly reduced comparing to the linear damping rate given by Eq. 1 after a possible formation of a flat plateau region at the distribution function. Estimations of the damping rate at the nonlinear stage of the Landau mechanism $\gamma^{\text {nl }}$ of a magnetosonic wave with the magnetic field amplitude $\delta B$ can be expressed as:

$$
\frac{\gamma_{\mathrm{ms}}^{\mathrm{nl}}}{\omega}=\frac{v_{\mathrm{coll}}}{\omega}\left(\frac{B_{0}}{\delta B}\right)^{3 / 2} \frac{\sin ^{1 / 2} \theta}{\cos ^{2} \theta}\left[\sqrt{\frac{2 m_{e}}{m_{p}}}+5 \exp \left(-\frac{v_{\mathrm{Ti}}^{2}}{v_{\mathrm{ph}}^{2} \cos ^{2} \theta}\right)\right]
$$

The effective particle collision rate $v_{\text {coll }}$ introduced into Eq.2 in a phemonological way is determined by the magnetic fluctuations at the background ion gyro-scale $\left(r_{\mathrm{gi}} \sim 10^{10} \mathrm{~cm}\right)$. In particular, the firehose and mirror instabilities at saturation in the marginal stability regime may provide $v_{\text {coll }} \sim \beta S_{l}$, where $S_{l}$ is the linear shear magnitude (Kunz et al, 2014).

Simulations by Matsumoto (1977) of nonlinear wave-particle interactions in a single sinusoidal magnetosonic mode of a finite amplitude demonstrated the test particle trapping, saturation, oscillation of the kinetic energy, and the diffusion of particles in velocity space. He has found that in a system without the Coulomb collisions the damping rate of the single magnetosonic wave is roughly $\propto \delta B^{-3 / 2}$ which is consistent with Eq. 2, 
A similar approach allows to estimate the Landau damping rate of Alfven mode in the non-linear regime of the distribution function flattening as

$$
\gamma_{\mathrm{a}}^{\mathrm{nl}} \sim \gamma_{\mathrm{ms}}^{\mathrm{nl}} \times\left(\frac{\omega}{\omega_{B}}\right)^{1 / 2}
$$

where $\omega_{B}$ is the gyrofrequency of a background ion, and for MHD waves the factor $\omega / \omega_{B}<1$. The difference between $\gamma_{\mathrm{a}}^{\mathrm{nl}}$ and $\gamma_{\mathrm{ms}}^{\mathrm{nl}}$ is due to the Alfven wave polarization.

In the case of the Alfven waves of relatively large amplitudes $\frac{\delta B}{B_{0}}>\frac{\omega}{\omega_{B}}$ the ion reflections on the magnetic field maxima of the wave provided the mode damping rate of

$$
\frac{\gamma_{\mathrm{a}}^{\mathrm{nl}}}{\omega} \approx \frac{1}{8 \pi^{3 / 2}}\left(\frac{\delta B}{B_{0}}\right)^{2} \frac{v_{\mathrm{Ti}}}{v_{\mathrm{a}}}\left[\sqrt{\frac{m_{e}}{m_{p}}}+\exp \left(-\frac{v_{\mathrm{Ti}}^{2}}{v_{\mathrm{a}}^{2}}\right)\right] .
$$

which may dominate over the non-linear Landau damping given by Eq. 3. At the same time the particle energization due to reflections contribute to the superthermal particles pool production. We shall discuss below in $\S 3$ the production of the superthermal particles by reflections at the shock waves.

The semi-qualitative analytic estimations discussed above were made for a simplified case of a single wave with well defined polarization, while in the ICM case we expect turbulent states with a broad range of interconnected scales. It means that the exact kinetic modeling should be performed with the selfconsistent particle-incell type simulations, which would allow to study the possible and likely departures of particle distribution function of the background plasma from the equilibrium Maxwellian distributions. This is very important for the accurate estimations of the non-thermal part of the plasma pressure in the clusters.

One should note that in the low $\beta$ plasmas of the warm and cold phases of the interstellar matter in galaxies the particles which are in Landau resonance with MHD waves have momenta at the declining part of the distribution function. Hence it is energetically feasible to build up the plateau in this regime to avoid the strong Landau damping. On the other hand it is not easy to build up the plateau in the hot interstellar plasma produced by supernovae (Bykov and Toptygin, 1983), where multiple weak shocks may dominate the turbulence (Bykov and Toptygin 1987). In the high $\beta$ plasma of a hot cluster it would be necessary to build up the plateau close to the peak of the distribution, which is energetically very demanding. If the departures from the Maxwellian ion distribution are present indeed, they can be constrained by the fine ion spectroscopy of the hot X-ray emitting plasma. Most likely these highly turbulent regions are not spread over the whole relatively quiet ICM but are intermittent and localized in the vicinity of the fast moving interacting structures.

Therefore in the cluster environment the collisionless compressible and incompressible modes of wavelengths $\lambda<\lambda_{C}$ are likely produced by fast motions of the relaxing cluster sub-structures, plasma instabilities and turbulent cascading of the large scale motions associated with shocks. The magnetic fluctuations of scales $\lambda \sim$ $10^{12}-10^{17} \mathrm{~cm}$ which are responsible for resonant scattering of GeV-TeV regime cosmic rays in the intercluster medium have scale sizes well below $\lambda_{C}$, but much larger than the thermal ion Larmor-scale. 
Hybrid-kinetic numerical simulations by Kunz et al (2014) revealed that momentum and heat transport in plasma structures with a persistent shear can be governed by magnetic fluctuations of $\delta B / B \sim 1$ at the ion- and sub-ion-Larmor-scales produced by firehose and mirror instabilities in a hot collisionless plasma in clusters of galaxies (see also Schekochihin et al, 2008; ZuHone et al, 2015; Komarov et al, 2016). The strong magnetic field fluctuations of the thermal ion Larmor-scale are important for the plasma heat conduction and viscosity, but they can not scatter efficiently CRs.

The very extended dynamical range of the fluctuation wavenumbers in the system makes the full scale particle-in-cell simulations to be unfeasible by now. In the meantime particle-in-cell simulations of the structure of collisionless shocks in high $\beta$ plasma can be successfully done given that much narrower dynamical range of scales are involved there. The simulations of collisionless shocks demonstrate clear departures from the Maxwellian distribution of ions which provide microscopic justification of the local effects of particle injection into Fermi acceleration on macroscopic scales of we shall discuss this in $\S 3$.

\section{Particle-in-cell modeling of collisionless shocks in hot cluster plasmas}

Shocks of different scales are playing a crucial role at different stages of cluster formation and evolution transforming the energy of the gas bulk motions to the plasma heating, turbulence production and particle acceleration. Collisionless shocks in clusters can locally produce a population of superthermal particles some fraction of which may be further accelerated/re-accelerated to long-lived relativistic component. Collisionless shock microphysics is essential at the stages of shock formation, shock dynamics, plasma heating and particle acceleration. Therefore the collisionless shock phenomenon is a multi-scale strong coupling non-linear problem in time and space (e.g. Marcowith et al, 2016). At the scales of some tens of the proton gyroradii particle-in-cell (PIC) simulations are the most efficient way to study the microphysical structure of the viscous velocity jump and plasma ion heating in high $\beta$ cluster plasmas (see e.g. Kato and Takabe, 2010).

Electron heating in the collisionless shocks is a widespread phenomena at all astrophysical scales from the bow shocks in the Earth and Saturn and the solar flares to supernova remnants and the cluster of galaxies scale. The electron heating efficiency (e.g. the postshock electron to proton temperature ratio) is very different for the collisionless shocks of different Mach numbers in the various environments (see e.g. Vink et al, 2015). The electron heating by shocks with Mach numbers $\mathscr{M} \sim 3$ (in the range suggested by observations of cluster radio shocks, see Van Weeren et al. this topical collection) in hot high $\beta$ plasma of clusters of galaxies were studied with particle-in-cell modeling by Guo et al (2017, 2018). They found that the electron whistler instability which can break the electron adiabatic invariance may occur due to the proton cyclotron and mirror modes accompanying the relaxation of proton temperature anisotropy. Then the non-adiabatic effects provide an efficient electron entropy production at the microscales before the Coulomb relaxation at much longer scales (Bykov et al, 2008b). 
Electrons can be directly injected to the non-thermal energies at the collisionless shocks to provide the primary leptonic component to be further energized in clusters of galaxies. Particle-in-cell simulations of the shocks of the sonic Mach numbers $\mathscr{M} \sim 3$ in the hot cluster plasmas were performed by Guo et al $(2014 \mathrm{a} b)$. They found that in quasi-perpendicular shocks about $15 \%$ of the initially thermal particles can be accelerated by the shock drift acceleration to a non-thermal power-law distribution of a slope about 2.4. Matsukiyo and Matsumoto (2015) found that the non-thermal electron acceleration by quasi-transverse shock is not explained merely by the shock drift acceleration, but the local wave- particle interactions can play an important role (see also Masters et al, 2017; Yang et al, 2018).

Combined hybrid particle-in-cell and test-particle simulations were used by Trotta and Burgess (2019) to model the influence of shock surface fluctuations on the acceleration of suprathermal electrons in three dimensional shock structure and to compare the $2 \mathrm{D}$ and $3 \mathrm{D}$ cases. The 3D structure of the shock front allowed a possibility for the electrons to interact with more than one surface fluctuation per interaction which may increase the electron energization. They also found that somewhat unrealistic electron trapping apparent in 2D simulations may cause the higher energization in the subcritical 2D shocks.

Contrary to the electron case the proton injection is efficient in quasi-parallel shocks and inefficient in the quasi-perpendicular geometry (Caprioli and Spitkovsky 2014). The microscopical process of proton injection in high beta plasmas can be studied by PIC simulations. Ha et al (2018) have recently simulated the reflection of incident protons and the self- excitation of plasma waves by CR-driven instabilities in the collisionless shocks under typical ICM conditions. They reported that only in ICM shocks with $\mathscr{M} \geq 2.25$ a sufficient fraction of incoming cosmic rays which are reflected at the shock (through a combination of the overshoot at the shock electric potential and magnetic mirror) produce further excitation of magnetic waves via CR streaming, hence starting diffusive shock acceleration. The simulated abundance of the merger shocks in the clusters is decreasing with the Mach number above $\mathscr{M} \geq 2.25$ (see e.g. Ryu et al, 2003b). Therefore, in this scenario only the relatively small fraction of merger shocks with $\mathscr{M} \geq 2.25$ might be able to inject cosmic rays in galaxy clusters, potentially alleviating the tension with $\gamma$-ray limits. The authors defined the injection fraction as the number of non-thermal ions with energies above 10 thermal proton energies in the shock downstream and found that the fraction ranges between $10^{-2}-10^{-3}$ for quasi-parallel shocks with $2.25 \leq \mathscr{M}<4$. The PIC simulations discussed above were performed for proton electron plasmas with the typical mass ratio $m_{p} / m_{e}=100$.

Apart from the radiation of the relativistic particles the non-thermal injection phenomena can be potentially constrained with the $\mathrm{X}$ ray spectroscopy (Kaastra et al. 2009). Therefore it is interesting to study the injection processes in multicomponent plasmas with account for at least some abundant ions in addition to protons and electrons. This would require the larger simulation domain and the longer run times than that in electron-proton plasmas. Hybrid simulations which treat ions kinetically while the electrons as a fluid are providing a way to extend the domain with the given computer resources. The hybrid codes (see e.g. Quest, 1988, Winske and Omidi, 1996 Giacalone et al, 1997; Lipatov, 2002, Gargaté et al, 2007; Caprioli and Spitkovsky 
2014; Kropotina et al, 2016) are widely used for modeling of collisionless shocks. We discuss in $\S 3.1$ the effect of $\mathrm{He}$ ions on the shock injection process and the shape of $\mathrm{He}$ and $\mathrm{Fe}$ ion distributions at the collisionless shocks in high $\beta$ ICM plasma.

\subsection{Hybrid simulations of shocks in hot multicomponent plasmas}

To address the effect of a realistic cluster plasma composition on the acceleration of CRs and the resulting departures of the ion heating from that given by the RankineHugoniot equations we present in this section some results of the hybrid simulations of shocks in high $\beta$ multi-ion cluster plasmas. The simulations discussed below were performed with the kinetic, three dimensional second order accurate, divergenceconserving hybrid code Maximus (Kropotina et al, 2018). Hybrid codes are a special class of kinetic codes which combine Vlasov-Maxwell equations for ions with hydrodynamic equations for electrons. The Vlasov equation for ions is solved within the Lagrangian approach, i.e. tracing the individual macro-particles in the continuous phase space. Each macro-particle represents the ensemble of real ones. The electrons are treated as a massless neutralizing fluid, which leads to the following equations system:

$$
\begin{aligned}
& \frac{d \mathbf{r}_{k}}{d t}=\mathbf{V}_{k} \\
& \frac{d \mathbf{V}_{k}}{d t}=\frac{Z_{k} e}{A_{k} m_{p}}\left(\mathbf{E}+\frac{1}{c} \mathbf{V}_{k} \times \mathbf{B}\right) \\
& \frac{\partial \mathbf{B}}{\partial t}=-c \nabla \times \mathbf{E} \\
& \mathbf{E}=\frac{1}{4 \pi \rho_{c}}(\nabla \times \mathbf{B}) \times \mathbf{B}-\frac{1}{c \rho_{c}}\left(\mathbf{j}_{\text {ions }} \times \mathbf{B}\right)-\nabla P_{e} / \rho_{c} \\
& \mathbf{j}_{\text {ions }}=\sum_{\text {cell }} S\left(\mathbf{r}_{k}\right) Z_{k} e V_{k} \\
& \rho_{c}=\sum_{\text {cell }} S\left(\mathbf{r}_{k}\right) Z_{k} e
\end{aligned}
$$

where $\mathbf{r}_{k}, \mathbf{V}_{k}, Z_{k}, m_{k}$ represent coordinates, velocities, charge and mass of macroparticles, $\rho_{c}, \mathbf{j}_{\text {ions }}$ - ions charge density and current, $P_{e}$ - electrons pressure, and $S\left(\mathbf{r}_{k}\right)$ - quadratic weighting function for charge deposit. Hereafter coordinates are given in units of proton inertial lengths $l_{i}=c / \omega_{p}$, velocities in alfvenic ones, and the mass density and magnetic field are normalized to their far upstream values. For the number density of ions, $n$, in multispecies plasma of mass density $\rho$ we took the proton concentration in the pure hydrogen plasma of the same $\rho$ (Matthews, 1994). The equation system must be closed by the electrons equation of state, which is actually unknown. The quasineutrality condition yields $n_{e}=n_{i} \equiv n$, so only the electrons downstream temperature is left to be determined to obtain $P_{e}$. The latter was taken from the PIC simulations with the code Tristan-MP (developed by: A.Spitkovsky, L.Gargate, J.Park, L.Sironi on the base of original TRISTAN code by O. Buneman.), and appeared to be consistent with the analytic estimations from (Vink et al, 2015), 
supposing 5\% energy exchange between electrons and ions, i.e. $T_{e}=T_{i}$ for $\mathscr{M}_{s}=2.0$ and $T_{e}=0.7 T_{i}$ for $\mathscr{M}_{s}=3.0$. The resulting equation system was solved numerically on a $3 \mathrm{~d}$ cartesian grid with 50 macro-particles per species per cell (ppsc) in the far upstream, which resulted in more than 150 ppsc downstream, due to the shock compression. The simulation box spanned $50,000 \times 1 \times 200$ cells of size $(0.5 \times 0.5 \times 0.5) l_{i}$.

The shock was initialized via the reflection of hot super-sonic super-alfvenic flow traveling in the negative $x$-direction from the conducting wall at the $x=0$ plane (the piston method). The upstream ratio of thermal to magnetic pressure $\beta$ was taken equal to 100 , with equipartition between electrons and ions. The far upstream magnetic field $B_{0}$ was placed in the $x-z$ plane and oriented obliquely to the flow, at a small angle $\theta=13^{\circ}$ to the shock normal $x$. In the simulation (i.e. downstream) frame the initial flow alfven Mach numbers were $\mathscr{M}_{a}=10$ and $\mathscr{M}_{a}=20$, which led to the sonic Mach numbers (in shock rest frame) equal to $\mathscr{M}_{s}=2.0$ and $\mathscr{M}_{s}=3.1$ respectively. Note that a substantial admixture of He would change the effective sonic Mach number.

The results of the hybrid simulations of the structure of the shock with the upstream parameters: $\mathscr{M} \approx 3$ (in the shock rest frame), $T_{e}=T_{i}=10^{8} \mathrm{~K}, \beta=100, \theta=$ $13^{\circ}$, solar abundance (75\% H II, $25 \% \mathrm{He} \mathrm{III,} 0.1 \%$ Fe XXVI by mass) are presented in Fig. 2 The structure is given at time moment $t=600 \Omega^{-1}$ after shock initialization.

Then in Fig. 3 we compare the spectra of protons obtained in the long run hybrid simulations to the PIC simulations by Ha et al (2018) at $t=90 \Omega^{-1}$. Far downstream proton energy spectra presented in this figure are for the same shock as in Fig. 2 at $t=150 \Omega^{-1}$ (gray curve) and $t=600 \Omega^{-1}$ (black curve). The corresponding spectrum of protons from Ha et al (2018) (run M3.2) at $t=90 \Omega^{-1}$ is shown by red dots. The consistency is apparent within the spatial and temporal variations of the power law tail.

In Fig. 4 far downstream energy distributions of protons (black), $\mathrm{He}^{+2}$ (blue) and $\mathrm{Fe}^{+25}$ (red) are shown for the same shock as that in Fig. 2 at $t=600 \Omega^{-1}$. The relaxation of the Fe ion is still in progress at this time. The distributions are normalized by unit, and the energy is given in terms of $E_{s h}=0.5 m_{p} V_{s h}^{2}=200\left[m_{p} V_{a}^{2}\right]$ (here $V_{s h}$ is in the simulation (i.e. downstream rest) frame).

The multi-ion composition of the ICM may have a prominent effect on both the temperatures and non-thermal tails of the ions passed through the collisionless shock and hence can be constrained with the X-ray spectroscopy of galaxy clusters. Theoretical models predicted an important role of the diffusion and sedimentation effects in the redistribution of elements in the hot interstellar and intracluster media (see e.g. Fabian and Pringle, 1977, Gilfanov and Syunyaev, 1984, Chuzhoy and Loeb, 2004. Ettori and Fabian, 2006; Medvedev et al, 2017, Biffi et al, 2018). X-ray observations of the galaxy clusters (see e.g. de Plaa et al, 2006; Böhringer and Werner, 2010, Mernier et al, 2017) provided clear evidences that the ICM does not have a primordial composition, but has been enriched with metals up to about 1.5 - 2 times of the solar abundance.

The helium abundance affects seriously the structure of electromagnetic fields in the shock transition region and the resulting ion distributions in the shock downstream. In Fig. 5 the far downstream energy distributions of $\mathrm{Fe}^{+25}$ ion are shown for different possible abundances of $\mathrm{He}^{+2}$ ions in the upstream flow. The solar abundance of $\mathrm{He}^{+2}$ results in the energy distribution of $\mathrm{Fe}^{+25}$ shown with the black curve, 


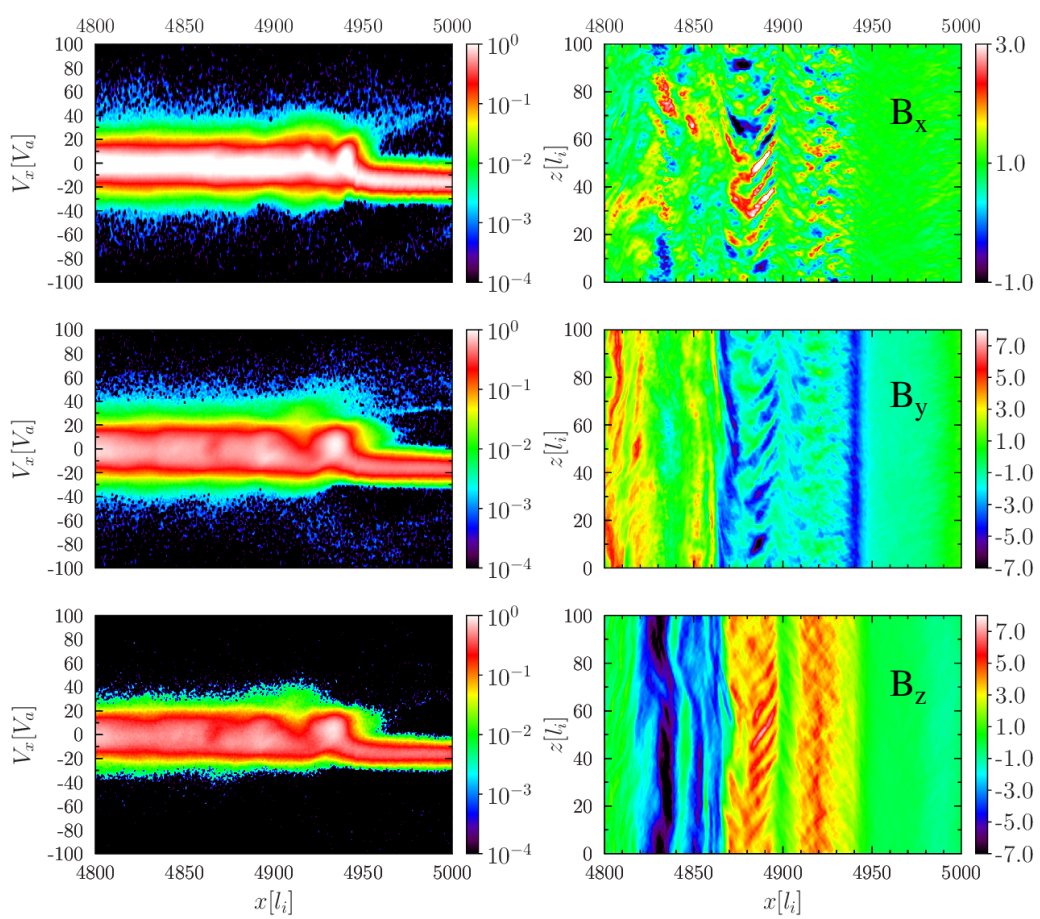

Fig. 2 Simulated structure of the shock with the following upstream parameters: $\mathscr{M} \approx 3$ (in the shock rest frame), $T_{e}=T_{i}=10^{8} \mathrm{~K}, \beta=100, \theta=13^{\circ}$, solar abundance $(75 \% \mathrm{H} \mathrm{II}, 25 \% \mathrm{He} \mathrm{III,} 0.1 \% \mathrm{Fe}$ XXVI by mass). The results are given at time $t=600 \Omega^{-1}$ after shock initialization. The corresponding upstream alfven Mach number in the simulation frame is $\mathscr{M}_{a}=20$. The $x-V_{x}$ phase spaces of protons, $\mathrm{He}^{+2}$ and $\mathrm{Fe}^{+25}$ are given in the left panel from top to bottom respectively, while the magnetic field components are color-coded in the right panel.

while the red curve is the $\mathrm{Fe}^{+25}$ distribultion in plasma with the doubled solar abundance of the helium. The next generation of X-ray observatories (e.g. Barret et al, 2018) may be used to constrain the helium abundance in clusters with the precise line spectroscopy of metals (e.g. $\mathrm{Fe}^{+25}$ ).

\subsection{The energy losses and the non-thermal tails}

While particle energy gain dominates over energy losses due to particle interactions in the vicinity of the shock of some thousands of gyroradii, at much larger distances the energy and momentum losses may become important.

The rate of energy losses $d E_{\mathrm{C}} / d t$ of a test ion of velocity $v$ due to the Coulomb collisions in fully ionized plasma of the electron temperature $T_{\mathrm{e}}$ can be approximated as (Schlickeiser, 2002) 


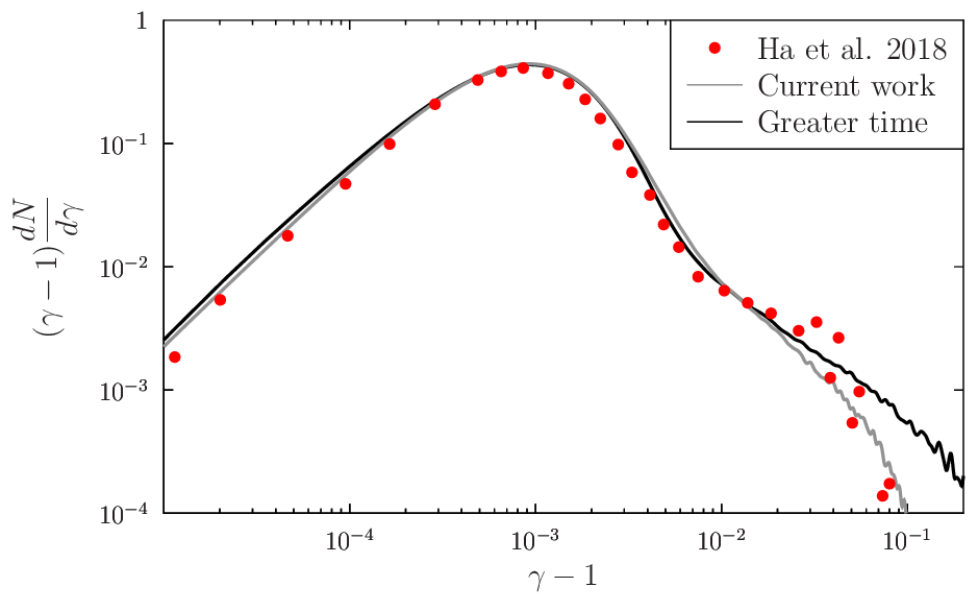

Fig. 3 Far downstream proton energy spectra for the same shock as in Fig. 2 at $t=150 \Omega^{-1}$ (gray curve) and $t=600 \Omega^{-1}$ (black curve). The corresponding spectrum from Ha et al (2018) (run M3.2) at $t=90 \Omega^{-1}$ is shown by red dots. The consistency is within the spatial and temporal variations of the power law tail.

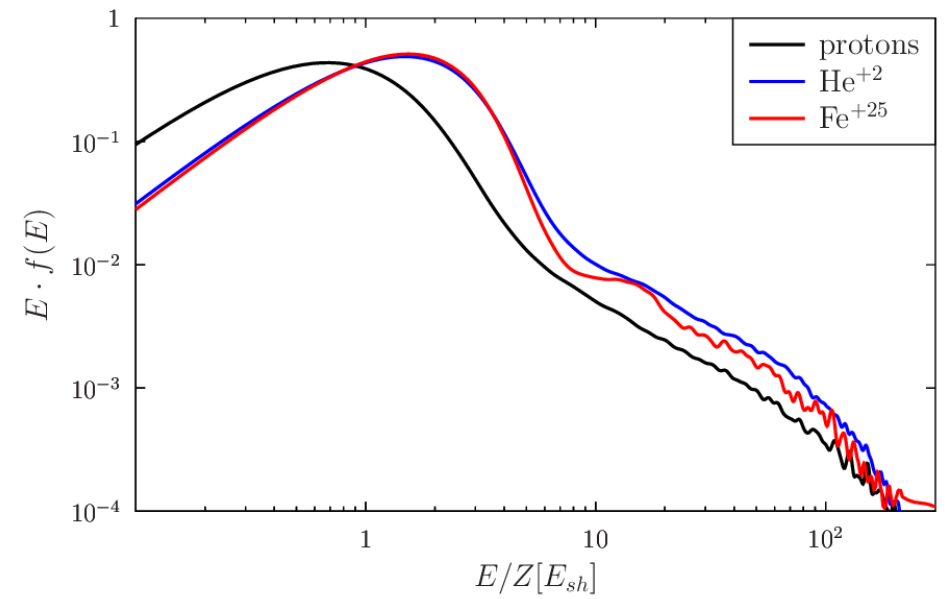

Fig. 4 Far downstream energy distributions of protons (black), $\mathrm{He}^{+2}$ (blue) and $\mathrm{Fe}^{+25}$ (red) for the same shock as that in Fig. 2 at $t=600 \Omega^{-1}$. The relaxation of Fe is still in progress at this time. The distributions are normalized by unit, and the energy is given in terms of $E_{s h}=0.5 m_{p} V_{s h}^{2}=200\left[m_{p} V_{a}^{2}\right]$ (here $V_{s h}$ is in the simulation (i.e. downstream rest) frame).

$$
-\frac{d E_{\mathrm{C}}}{d t}=3.1 \times 10^{-7} n_{\mathrm{e}} Z^{2} \frac{(v / c)^{2}}{\left(x_{m}\right)^{3}+(v / c)^{3}} \mathrm{eV} \mathrm{s}^{-1}
$$

where $x_{m} \approx 0.03\left(T_{\mathrm{e}} / 2 \times 10^{6} \mathrm{~K}\right)^{1 / 2}$.

At non-relativistic ion energies the lifetime of nuclei against the energy losses due to the inelastic Coulomb collisions in the fully ionized hot ICM can be obtained 


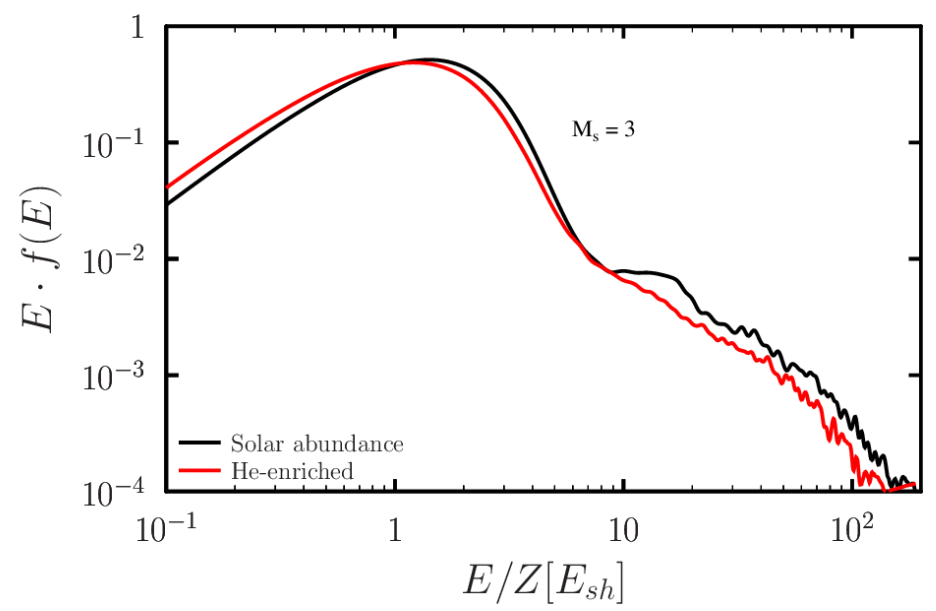

Fig. 5 Far downstream energy distributions of $\mathrm{Fe}^{+25}$ for different local abundances of $\mathrm{He}^{+2}$ in the intercluster medium. The energy distributions of $\mathrm{Fe}^{+25}$ for the solar (black) and for double (red) abundances of $\mathrm{He}^{+2}$ simulated for the same shock at $t=600 \Omega^{-1}$. The energy is given in terms of $E_{s h}=0.5 m_{p} V_{s h}^{2}=$ $200\left[m_{p} V_{a}^{2}\right]$ (here $V_{s h}$ is in the downstream rest frame).

by the integration of Eq. 11 The lifetimes $\tau_{\mathrm{s}}$ for $\mathrm{s}=\mathrm{p}, \mathrm{He}^{+2}$ and $\mathrm{Fe}^{+25}$ are shown in Fig. 6. The inelastic nuclear collisions provide a fragmentation of $\mathrm{He}, \mathrm{Fe}$ and the other nuclei even at the $\mathrm{MeV}$ energy regime with a characteristic lifetime longer than that is due to the Coulomb losses. Above the pion production threshold the energy losses are dominated by this process and the lifetime is limited to below $10^{17} \mathrm{~s}$ in typical hot ICM conditions. The energy losses of electrons in the ICM where the synchrotron and inverse-Compton processes are important were discussed earlier in Petrosian and Bykov (see e.g. 2008) .

An important parameter is the width $\left(\Delta_{\mathrm{S}}\right)$ of the region around the CR injector/accelerator (e.g. shock) filled with injected CRs. It can be estimated as $\Delta_{\mathrm{s}} \sim$ $\sqrt{2 v \tau_{\mathrm{s}} \Lambda}$ (where $\Lambda$ is the particle mean free path) assuming the diffusive propagation of CRs in the cluster. If $\Delta_{\mathrm{s}}$ is larger than the mean distance between the injectors than apart from the individual shocks of $\mathscr{M} \geq 2.25$ also large scale plasma motions and the weaker shocks can re-accelerate the seed population of the super-thermal particles. For injected particles of energy above $10 \mathrm{E}_{\mathrm{sh}}$ the mean free path due to the elastic Coulomb scatterings on the fully ionized ICM is above a Mpc, which mean that they would spread over the most of the ICM. However, if a magnetic turbulence of scales well below a parsec has a sizeable filling factor in the ICM, then the mean free path $\Lambda$ will be much smaller there and the Coulomb losses would be a factor. On the other hand the magnetic turbulence providing efficient scattering of non-thermal particles would allow CR re-acceleration by the long-wavelength compressible plasma motions of sub-Mpc scales produced by the merging/accretion processes. We shall discuss the processes now.

Apart from particle injection and acceleration by the individual shocks the large scale plasma motions and weaker shocks may re-accelerate particles from the long- 


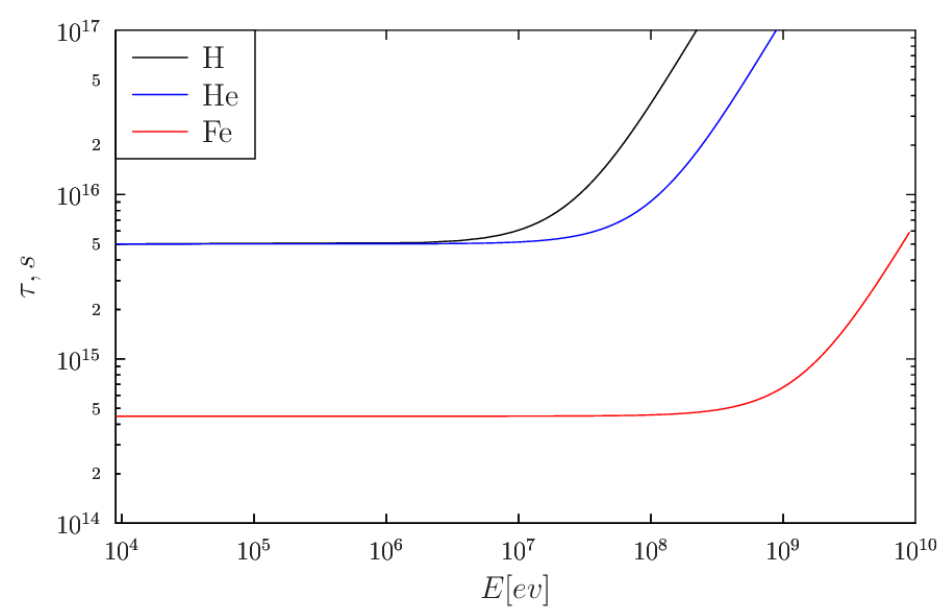

Fig. 6 The lifetime of nuclei against the energy losses (the Coulomb losses in the fully ionized ICM) protons (black), $\mathrm{He}^{+2}$ (blue) and $\mathrm{Fe}^{+25}$ (red). The lifetime at the higher energies is limited by the inelastic nuclear interaction processes at about $10^{17} \mathrm{~s}$.

lived pool of non-thermal particles. In $\S 4.1$ we discuss the spectra of the nonthermal particles re-accelerated by the multiple shocks and the associated compressible plasma motions.

\section{Particle acceleration by ensemble of shocks in clusters of galaxies}

The accretion and merging processes, as well as fast supersonic motions of dark matter halos with baryonic component should launch shocks in the ICM, from a megaparsec scale down to about $10 \mathrm{kpcs}$, where Coulomb collisions are still important. The kinetic energy of large scale motions is a huge reservoir of power of the acceleration of particles and magnetic field amplification. However, particle acceleration by the Fermi mechanism, which allows to transfer a part of the energy of the plasma motions to relativistic particles, can only proceed in presence of effective particle scatterings by magnetic fluctuations. This can be provided in the presence of magnetic fluctuations of scales comparable to the gyroradii of the accelerated particles. In the typical ICM magnetic fields $(\sim \mu \mathrm{G})$ the scales of the resonant fluctuations are below $10^{17} \mathrm{~cm}$, i.e. well below the energy containing scale of the plasma motions.

If the magnetic fluctuations of scales $l$ in the range $10^{12}-10^{17} \mathrm{~cm}$ exist in the collisionless regime and are a part of the extended Kolmogorov type turbulent spectrum of spectral index $v$ with the energy containing scale $L$, then the mean free path of CR particles can be estimated from the quasi-linear theory as $\Lambda(p) \sim g(v) L\left(r_{g} / L\right)^{2-v}$ where $g(v)$ is a numerical factor (see e.g. Toptygin 1985). Then the parameter $\eta(p)$ can be expressed as

$$
\eta(p)=u L / v \Lambda(p)=u / c \times g^{-1}(v)\left(L / r_{g}\right)^{2-v}
$$


For a typical bulk motions of the velocity amplitude $u \gtrsim 1,000 \mathrm{~km} / \mathrm{s}$ at $\geq 100$ kpcs scales, and with the extended Kolmogorov type magnetic turbulence, one has $\eta(p)>1$. For cosmic rays of low enough energies to satisfy $\eta(p)>1$, the CR distribution function $F(r, p, t)$ is highly intermittent in space, with sharp concentration gradients in the vicinity of the shocks and the smooth backround distribution in between of the shocks. Contrary to the case of the low energy CRs with $\eta(p)>1$, in the high energy regime the typical distance between the shocks is $L<l_{\mathrm{sh}}(p)=c / u \times \Lambda(p)$ which is characterizing the width the distribution of CR of momentum $p$ in the shock vicinity. Therefore the condition of $\eta(p)<1$ provides a smooth $\mathrm{CR}$ distribution at the high energy end of the accelerated particles.

Since the particle distribution within ICM with the shock ensemble is highly intermittent at low $\mathrm{CR}$ energies ( $\mathrm{GeV}-\mathrm{TeV}$ energies which we are interested in), the statistical description of the nonthermal particle distributions is different from that of the statistically-homogeneous systems (Bykov and Toptygin, 1990). They decomposed the distribution function in two parts $F(r, p, t)=N(r, p, t)+\delta N(r, p, t)$, where as usually $\langle F(r, p, t)\rangle=N(r, p, t)$ is the distribution function averaged over the ensemble of the large scale fluctuations. Then at the high energy end $\eta(p)<1$ provides $N(r, p, t)>>|\delta N(r, p, t)|$ and the standard quasi-linear procedure of averaging is appropriate. For the low energy CRs where $\eta(p)>1$ and the perturbation $|\delta N(r, p, t)|$ is not small anymore at the shock fronts the perturbation theory fails, but one can use another approximation. In this case one can use a solution of the diffusion-advection equation which connects the particle distribution function at a given shock surface $F_{\mathrm{i}}(\mathrm{r}, p, t)$ with the distribution function in the far upstream of the shock which can be approximated by the average distribution function $N(r, p, t)$.

$$
F_{\mathrm{i}}(\mathrm{r}, p, t)=\theta\left(z_{\mathrm{i}}\right) \Phi_{\mathrm{i}}+\theta\left(-z_{\mathrm{i}}\right)\left[\Phi_{\mathrm{i}} \exp \left(\Delta u_{\mathrm{i}} z_{\mathrm{i}} / \chi_{\mathrm{i}} z_{\mathrm{i}}\right)+N\left(z_{\mathrm{i}}, p\right)\right]
$$

where $\theta\left(z_{\mathrm{i}}\right)$ is the Heaviside step function of a coordinate $z_{\mathrm{i}}$ along the normal to the plane of the where i-th shock surface with the flow velocity jump $\Delta u_{\mathrm{i}}$ and the particle diffusion coefficient in the shock upstream $\chi_{\mathrm{i}}$. The function $\Phi_{\mathrm{i}}$ depends on the particle power law distribution of index $\gamma_{i}$ which is formed by the diffusion shock acceleration:

$$
\Phi_{\mathrm{i}}(\mathrm{r}, p, t)=\left(2+\gamma_{\mathrm{i}}\right) p^{-\left(2+\gamma_{\mathrm{i}}\right)} \int_{0}^{p} \mathrm{~d} p^{\prime} p^{\left(\gamma_{\mathrm{i}}+1\right)} N\left(p^{\prime}\right) .
$$

In the simplest case $\gamma_{\mathrm{i}}=\left(\sigma_{\mathrm{i}}+2\right) /\left(\sigma_{\mathrm{i}}-1\right)$ is determined by the shock compression ratio $\sigma_{\mathrm{i}}$, while some other effects like the Alfvenic drift (see e.g. Kang and Ryu. 2018) can be important and will .

In this approximation Bykov and Toptygin (1990) obtained the following system of equations for the distribution function $N(r, p, t)$ averaged over the ensemble of large scale plasma motions with frozen-in magnetic field fluctuations of a broad scale range and shocks:

$$
\frac{\partial N}{\partial t}-\frac{\partial}{\partial r_{\alpha}} \chi_{\alpha \beta} \frac{\partial N}{\partial r_{\beta}}=G \hat{L} N+\frac{1}{p^{2}} \frac{\partial}{\partial p}\left(p^{4} D-p^{2} \dot{p}_{L}\right) \frac{\partial N}{\partial p}+A \hat{L}^{2} N+2 B \hat{L} \hat{P} N+Q_{j}(p),
$$


The operators $\hat{P}$ and $\hat{L}$ are given by the equations

$$
\hat{P}=\frac{p}{3} \frac{\partial}{\partial p} ; \quad \hat{L}=\frac{1}{3 p^{2}} \frac{\partial}{\partial p} p^{3-\gamma} \int_{0}^{p} \mathrm{~d} p^{\prime} p^{\prime \gamma} \frac{\partial}{\partial p^{\prime}} .
$$

The source term $Q_{j}(r, p, t)$ is determined by the properly averaged injection rate of the nuclei of a type $j$, while $\dot{p}_{L}(p)$ is the rate of particle energy losses. The kinetic coefficients $A, B, D, \tau_{s h}$, and $\chi_{\alpha \beta}$ are expressed in terms of the spectral functions which describe correlations between large scale turbulent motions and shocks.

We presented here a simplified version of the kinetic equations assuming that all of the $\gamma_{i}=\gamma$ which is the case either for the ensemble of strong shocks (where the indexes are close to 2), or for the shocks of identical strengths. The general case of shock ensemble is described by equations presented in Bykov and Toptygin (1990 1993).

The kinetic coefficients satisfy the following renormalization equations (assuming a quai-isotropic turbulent field):

$$
\begin{gathered}
\chi=\kappa+\frac{1}{3} \int \frac{d^{3} \mathbf{k} d \omega}{(2 \pi)^{4}}\left[\frac{2 T+S}{i \omega+k^{2} \chi}-\frac{2 k^{2} \chi S}{\left(i \omega+k^{2} \chi\right)^{2}}\right], \\
D=\frac{\chi}{9} \int \frac{d^{3} \mathbf{k} d \omega}{(2 \pi)^{4}} \frac{k^{4} S(k, \omega, t)}{\omega^{2}+k^{4} \chi^{2}}, \\
A=\chi \int \frac{d^{3} \mathbf{k} d \omega}{(2 \pi)^{4}} \frac{k^{4} \tilde{\phi}(k, \omega, t)}{\omega^{2}+k^{4} \chi^{2}}, \\
B=\chi \int \frac{d^{3} \mathbf{k} d \omega}{(2 \pi)^{4}} \frac{k^{4} \tilde{\mu}(k, \omega, t)}{\omega^{2}+k^{4} \chi^{2}} .
\end{gathered}
$$

Here $T(k, \omega, t)$ and $S(k, \omega, t)$ are the transverse and the longitudinal parts of the Fourier components of the turbulent velocity correlation tensors. The time dependence is determining the slow evolution of the spectra on the time intervals longer $t>\omega^{-1} . G=\left(1 / \tau_{s h}+B\right)$ are determined by the shock statistics (see e.g. Bykov and Toptygin, 1987, Ryu et al, 2003b). Correlations of the velocity jumps at shock fronts are described by $\tilde{\phi}(k, \omega, t)$, while $\tilde{\mu}(k, \omega, t)$ represents the correlations of shocks and long wavelength rarefaction waves. The introduction of these spectral functions is required due to the intermittent character of the system with multiple shocks. The local diffusion coefficient $\kappa(p)=v \Lambda(p) / 3$ is due to the CR scattering by short scale magnetic fluctuations. At low energy regime under consideration where $\eta(p)>1$ the local diffusion coefficient $\kappa(p)$ is smaller than $\chi$, and the global transport of the low energy CRs is governed by the turbulent diffision.

As it is seen from Eq 18 in the case of Fermi acceleration of CRs by the long wavelength MHD turbulence (where the energy containing scale size of the turbulence $L$ is larger than the CR mean free path $\Lambda(p)$ ) the compressible longitudinal part of the spectrum dominates the acceleration Bykov and Toptygin (see for review 1993). Therefore the Fermi type acceleration of CRs by the longitudinal large scale MHD motions result in a damping of the turbulent motions if the CR population is numerous enough. On the other hand the turbulent diffusion of CRs in the large scale 
MHD turbulence as it follows from Eq. 17 is provided by both the incompressible vortex type motions (described by the transverse part of the spectrum $T(k, \omega, t)$ ) and the compressible turbulence (longitudinal part of the spectrum $S(k, \omega, t)$ ).

\subsection{Spectra of cosmic rays accelerated by ensemble of shocks}

The kinetic energy of the baryonic component of a large cluster of galaxies can be as large as $\sim 10^{63}$ ergs and the kinetic power release within the intercluster meduim may reach $10^{47} \mathrm{erg} \mathrm{s}^{-1}$ during major merger events. Vortex electric fields generated by the large scale motions of highly conductive plasma with shocks result in a non-thermal distribution of the charged particles. This may transfer some fraction of the kinetic power of the baryonic matter into the non-thermal components. The superthermal particles both freshly injected at shocks and the long lived pool produced by the previous substructure mergers, AGN activity and the other processes in clusters of galaxies can be reaccelerated by the large scale MHD motions of magnetized plasma with shocks.

We can apply the kinetic equation Eq. (15) described above to model the efficiency of the conversion of the kinetic power of the compressible supersonic turbulence to $\mathrm{CR}$ production. The model considers a simplified statistically homogeneous system, which only consists of the large-scale turbulent plasma motions and CRs, and the total energy of the system is conserved. In the model, the spectra of turbulence were parameterized in the following way:

$$
\psi(k, \omega, t)=\psi(k, t) \frac{\Gamma_{k}}{2}\left[\frac{1}{\left(\omega-\omega_{0}\right)^{2}+\left(\Gamma_{k}^{2} / 4\right)}+\frac{1}{\left(\omega+\omega_{0}\right)^{2}+\left(\Gamma_{k}^{2} / 4\right)}\right] .
$$

Here we introduced $\psi(k, \omega, t)=(T(k, \omega, t), S(k, \omega, t)), \Gamma_{k}=\tau_{c}(k)^{-1}, \omega_{0}(k)=v_{\mathrm{ph}} k$, $S(k, t) \propto S_{0}(t) e^{-\frac{k^{2}}{k_{0}^{2}}}$, where $k_{0}=2 \pi L^{-1}$

In Fig. 7] we illustrate the simulated temporal evolution of the spectra of CRs accelerated in a cluster of about $2 \mathrm{Mpc}$ scale size with the amplitude of the bulk velocity fluctuations $\sim 2,000 \mathrm{~km} / \mathrm{s}$ (i.e. $\sqrt{S(0) k_{0}} \sim 2,000 \mathrm{~km} / \mathrm{s}$ ) and the energy containing scale $L=100 \mathrm{kpc}$. In this case we assumed a reacceleration scenario where CRs had initially a narrow energy spectrum with the energy density of about $10^{-4}$ (left panel) or $10^{-3}$ (right panel) of that of the turbulence. The conversion efficiency could exceed $10 \%$. The asymptotic CR energy spectra at the time moments after $3 \tau_{\mathrm{a}}$, where $\tau_{\mathrm{a}}=9 L / u$ are approaching the power-law with $N(p) p^{2} \propto p^{-a}$ (blue curves). The asymptotic spectra are harder in case of the lower injection rate. At earlier time moments $0.3 \tau_{\mathrm{a}}$ (red curves) and $1 \tau_{\mathrm{a}}$ (magenta curves) one can see some spectral evolution from soft to hard and then asymptotically to the power-law spectra. The model is simplified, yet it can provide an illustration of the possible role of non-linear feedback on the spectral evolution. More exact models must account for the evolution of the short scale collisionless turbulence which provide the $\mathrm{CR}$ scattering $(\Lambda(p)$ was fixed here) and a consistent model of CR injection and gas heating by shocks as it was discussed in $\$ 3$. 


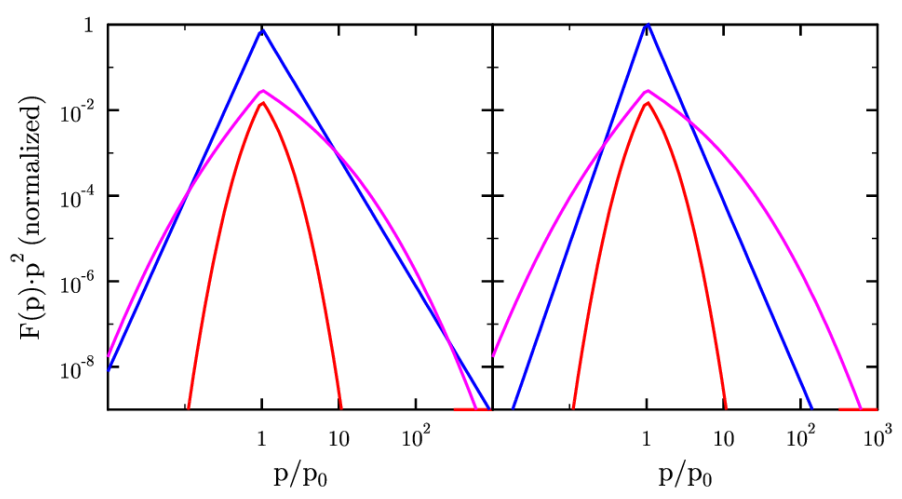

Fig. 7 The temporal evolution of the nonthermal protons for a cluster of a size $\sim 2 \mathrm{Mpc}$ with the large scale turbulence of $L \sim 100 \mathrm{kpc}$ and velocity magnitude $2 \times 10^{3} \mathrm{~km} / \mathrm{s}$. CRs were injected at $p=p_{0}$ with the energy density about $10^{-4}$ (left panel) and $10^{-3}$ (right panel) of that of the turbulence. The spectra are shown at the time moments $-0.3 \tau_{\mathrm{a}}$ (red curves), $1 \tau_{\mathrm{a}}$ (magenta curves) and the asymptotic spectra after 3 $\tau_{\mathrm{a}}$ (blue curves).

\subsection{Particle acceleration by multiple shocks in the heliosphere}

Cosmic ray acceleration by the ensemble of shocks and long wavelength MHD motions are likely to occur in other astrophysical objects. Direct measurements of both magnetic turbulence and non-thermal particles are available in the heliosphere. The analysis of the suprathermal spectra in the interplanetary medium observed in the quiet periods revealed the spectra $f(v) \propto v^{-5}$ (Fisk and Gloeckler, 2006, 2014, 2017). The spectra are consistent with the model for the intercluster medium $N(p) p^{2} \propto p^{-3}$ as in the left panel in Fig. 7 (see also Fig. 2 in (Bykov, 2001)). The origin of the spectra observed in the interplanetary medium is associated with particle acceleration by the compressional plasma wave trains consisting of a series of compression or rarefaction regions (Fisk and Gloeckler, 2006, Zhang, 2010, Jokipii and Lee, 2010, Antecki et al, 2013; Fisk and Gloeckler, 2017) which can be described by Eqs. (15)(20) and spectra given in Fig. 7. It is important that in this case observations revealed the extended spectrum of magnetic fluctuations which provide effective scattering of non-thermal particles.

\subsection{Particle acceleration by multiple shocks in galactic superbubbles}

Energetic particles likely comprise a long-lived CR component which may contain a sizeable fraction of the kinetic energy released by the winds and supernova ejecta in galactic superbubbles (Bykov, 2001, 2014). Strong supernova shocks accompanied by the multiple secondary weak shocks may be the responsible for the observed excess of gamma-ray emission detected by Fermi-LAT in the Cygnus OB2 associ- 
ation (Ackermann et al, 2011) and in the star forming region G25.0+0.0 (Katsuta et al 2017). The observed gamma-ray spectra roughly correspond to CR distribution $N(p) p^{2} \propto p^{-2}$. The hard spectra are expected to be the case in the systems with strong shocks like those produced by supernovae (Bykov, 2001, 2014, Grenier et al, 2015; Lingenfelter, 2017, Bykov et al, 2018). In the clusters of galaxies with CR acceleration by weak shocks and long-wavelength turbulence in the model discussed above the spectra are softer with $N(p) p^{2} \propto p^{-3}$ if the broad band of magnetic fluctuations providing the effective $\mathrm{CR}$ scattering is available in the intercluster medium. However, if the scattering of CRs in GeV-TeV regime is inefficient and $\Lambda(p)$ is long enough to provide $\eta(p)<<1$, then the CR acceleration time is getting longer than their escape time and the spectra are steeper. Observations of the non-thermal emission (see e.g. Ferrari et al, 2008, Feretti et al, 2012; Brunetti and Jones, 2014 Prokhorov and Churazov, 2014, Zandanel et al, 2015) as well as the future high resolution X-ray spectroscopy (Kaastra et al, 2009) can constrain the particle acceleration scenario and the contribution of the non-thermal components to the energy budget of the clusters.

Apart from the diffusive CRs propagation in clusters of galaxies and the diffusive shock acceleration which is considered as a rather realistic mechanism of the efficient acceleration of non-thermal particles in clusters of galaxies the superdiffusive propagation of CRs may also be essential under some conditions in both accretion and merger shocks (see e.g. Bykov et al, 2017, Zimbardo and Perri, 2018) and the magnetic reconnection process was discussed in this context by Lazarian and Brunetti (2011).

\section{Cosmological simulations of cosmic ray acceleration and their problems}

Because of the expected complexity of the shock network in the dynamical environment of galaxy clusters, following the spectral and spatial evolution of accelerated $\mathrm{CRs}$ in the intracluster medium is a nontrivial task. The acceleration of cosmic ray electrons and of cosmic ray protons in cosmology generally poses very different challenges to numerical simulations, and for this reason the two processes are usually followed by means of two complementary approaches.

\subsection{CR electrons in cosmological simulations}

Unless they get (re)accelerated multiple times, cosmic ray electrons are expected to be short lived in typical ICM condition $\left(\leq 10^{8}\right.$ yr for radio emitting $\gamma \sim 10^{4}$ electrons, e.g. Van Weeren et al. this topical collection). Once accelerated, they cannot thus be advected nor diffuse more than $\sim 100 \mathrm{kpc}$ away from their injection site, before they loose most of their energy via synchrotron and Inverse Compton radiation. Given the energy budget which can be derived by radio observations, their dynamical impact on the ICM is also believed to be entirely negligible.

These circumstances justify the simple post processing treatment of CR electrons in most cases: typically once that a cosmological simulation is run, shock waves 
are detected using different shock finder methods, and the budget of CR electrons accelerated in the shock downstream is computed based on the information of a single timestep, assuming a quasi-stationary balance of DSA acceleration and radiative losses (e.g. Hoeft and Brüggen, 2007).

Very first works that studied the acceleration and energy evolution of electrons in the test particle regime, employed Eulerian schemes to model shocks and compute non-thermal emission from electrons (e.g. Miniati et al, 2001b; Miniati, 2003). Later on high resolution simulations employing smoothed-particle hydrodynamics (Keshet et al, 2004; Hoeft et al, 2008, Pfrommer et al, 2008; Battaglia et al, 2009. Araya-Melo et al, 2012; Nuza et al, 2012) or adaptive mesh refinement (Vazza et al 2010; Skillman et al, 2011; Vazza et al, 2012b; (Planelles et al, 2018) modelled the radio emission from shock-accelerated electrons as well, assuming DSA acceleration and ad-hoc prescriptions on ICM magnetic fields in a post-processing step. A few examples of non-thermal emission from shock-accelerated electrons in cosmological simulations are given in Fig 8

The most direct testbed for cosmological simulations of electron acceleration is represented by the fast growing class of radio relics sources (see Van Weeren et al., this topical collection).

In this respect, cosmological simulations can in general produce simulated radio relics that are similar in shape, total power and spectral index to those observed in radio relics, suggesting that at first order the typical kinetic power from merger shocks in simulations is realistically the main source to power radio emitting electrons (Hoeft et al, 2008, Battaglia et al, 2009, Skillman et al, 2011, Bonafede et al, 2012, Vazza et al 2012b; Hong et al, 2015). Also the relation between the observed relic power and the host cluster mass or luminosity is reasonably close to observations (Skillman et al, 2011, Nuza et al, 2012, de Gasperin et al, 2014, Nuza et al, 2017). However, once large samples of observed and simulated radio relics are compared using the same analysis pipeline, important differences emerge in the distribution of their morphological parameters (Nuza et al, 2017): in particular simulations tend to find more "small" and roundish relics than observations, and simulated relics are found on average to be less extended (by $\sim 15 \%$ ) and less distant (by $\sim 30 \%$ ) from the cluster centre than their real counterparts in the NVSS survey (see left panel of Fig 9). This may indicate that non-gravitational processes, like radiative cooling and energy feedback from galaxy evolution processes (not included in most simulations investigating radio relic emission), may affect the large-scale properties of radio emitting shocks as they sweep through the ICM.

The "microphysical" uncertainties in simulations are still very large, and mostly reflect the complexity of plasma processes reviewed in the previous Sections. Simulations are bound to guess the acceleration efficiency by shocks in the $\mathscr{M} \leq 2-5$ regime, based on DSA or its extrapolation, and in the literature typical values from a few percent to $\sim 10^{-5}$ of the shock kinetic energy are quoted to produce realistic radio power from merger shocks. It shall be noticed that acceleration efficiencies $\geq 10^{-3}$ poses problem to DSA as they imply an energy ratio of electron to proton at odds with basics expectations (e.g. Vazza and Brüggen, 2014, Brunetti and Jones 2014, see also next Section). 
Second, some degree of electron re-acceleration by shocks seems to be often necessary to explain radio emission from shocks with $\mathscr{M} \leq 2$ (see Van Weeren et al. this topical collection), which is larger than that from simple DSA (See right panel of Fig.9. This calls for the modelling of older populations of electrons which may get re-accelerated by shocks coincident with relics (e.g. Pinzke et al, 2013), and whose radio contribution may easily dominate over the one by "freshly injected" electrons for $\mathscr{M} \leq 4$ shocks (Kang et al, 2012b). Even more numerically challenging is the inclusion of turbulent re-acceleration of CR electrons in galaxy clusters (Donnert et al, 2013; Donnert and Brunetti, 2014) which has so far never been obtained for fully cosmological simulations.

Finally, only a few cosmological simulations to date could model the acceleration of relativistic electrons by shocks also including magnetic fields at run time, via MHD computations. Skillman et al (2013) presented the first MHD grid simulation of radio relic emission in a galaxy cluster simulated at high resolution with Adaptive Mesh Refinement in the ENZO code, reporting a high degree of polarization, tentatively in agreement with observations available at the time, as well as the evidence of a complex distribution of Mach numbers (and injection spectral indexes) at merger shocks (lower left panel in Fig 8). Wittor et al (2017) also recently used ENZO-MHD simulations and AMR to assess the distribution of shock obliquities in merger shocks (lower right panel in Fig.8), and studied the effect of a revised acceleration efficiency which get maximum for electrons at quasi-perpendicular shocks, as suggested by PIC simulations (Guo et al, 2014a), and in relation to the lack of hadronic emission from accelerated CR protons in galaxy clusters (see next Section).

\subsection{CR protons in cosmological simulations}

Different than in the case of CR electrons, the simulation of CR protons in cosmology is bound to follow the acceleration and advection of particles in a more self-consistent way and at run-time, due to the very large $(\sim 1-10 \mathrm{Gyr})$ lifetime of injected CRs. Several methods (mostly based on variations of "two-fluids models) have been developed over the last decade in order to couple the simulated evolution of large-scale structures to the accumulation of cosmic rays injected by cosmic shocks over time. While a few seminal works first explored the evolution of cosmic rays in cosmological grid simulations, neglecting the dynamical feedback of cosmic rays onto baryons (Miniati et al, 2001a; Ryu et al, 2003a; Miniati, 2003), the first high-resolution simulations incorporating the dynamical impact of cosmic rays were based on smoothedparticle hydrodynamics (Pfrommer et al, 2007, Enßlin et al, 2007; Jubelgas et al, 2008; Pfrommer et al, 2008, Pinzke and Pfrommer, 2010).

The above works have led to the first, quantitative predictions of the expected radial distribution of cosmic ray pressure relative to the gas pressure (see Fig 10], as well as to the predictions on the level of $\gamma$-rays and radio emission from the hadronic mechanism. Cosmic rays accelerated by structure formation shocks were suggested to be volume filling, capable to significantly affect the gas dynamics in the cluster volume, and potentially responsible for the diffuse radio emission in the form of radio 

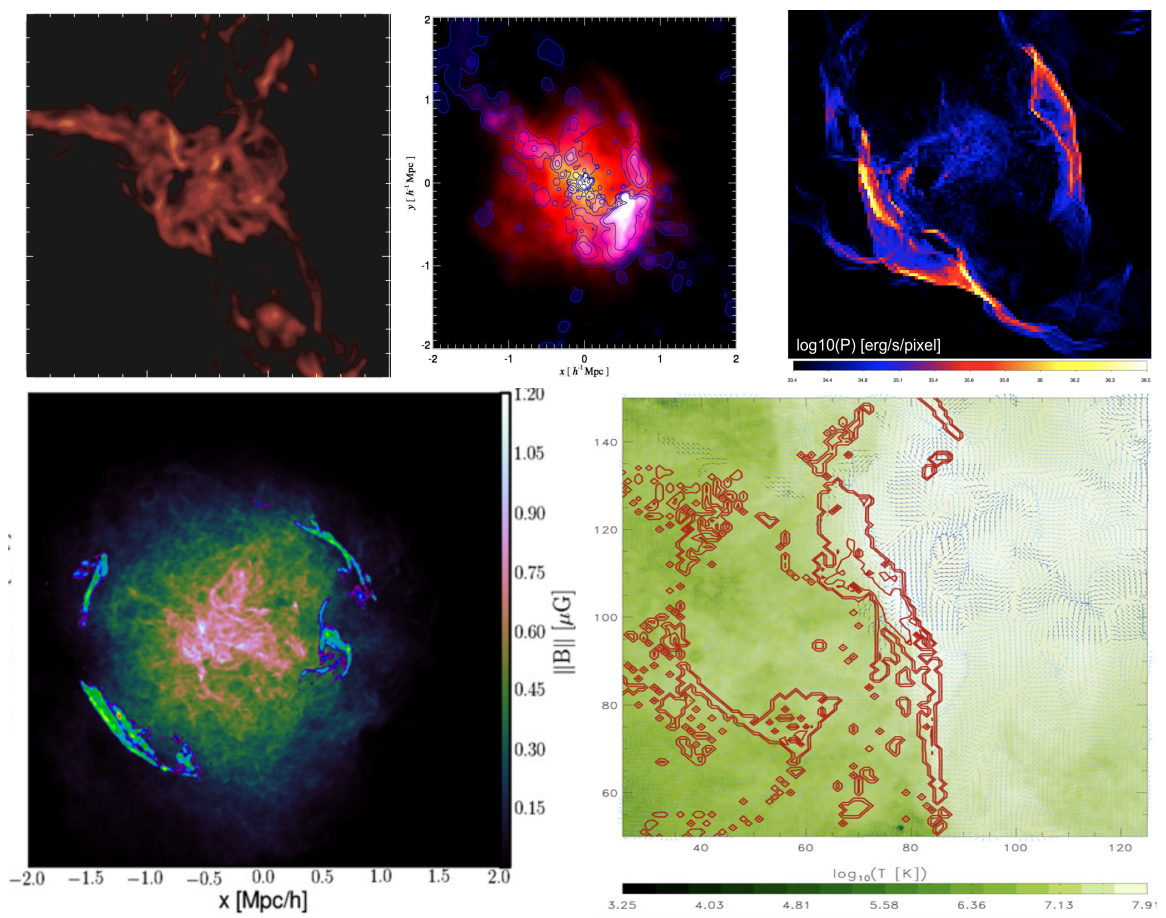

Fig. 8 Examples of simulated non-thermal emission from shock accelerated electrons in cosmological simulations. Top left panel: Hard-X emission $(\geq 100 \mathrm{keV})$ from the Inverse Compton scattering of electrons injected by cosmic shocks around a galaxy clusters, simulated with a fixed mesh simulation by Miniati (2003). Top central panel: primary radio emission at $150 \mathrm{MHz}$ (the contours show the emission detectable above $7 \cdot 10^{-4} \mathrm{mJy} / \mathrm{arcmin}^{2}$ ) in an SPH simulation by Battaglia et al (2009), with a postprocessing magnetic field which scales with thermal gas energy. Top right panel: radio emission from shock-accelerated emission in an Eulerian AMR simulation of a galaxy clusters (the image is $5 \mathrm{Mpc} / \mathrm{h}$ across) by Vazza et al (2012b), for an assumed flat magnetic field of $5 \mu G$ everywhere. Bottom left panel: simulated radio emission at $1.4 \mathrm{GHz}$ from shock-accelerated electrons (bright colors) in the Eulerian AMR simulation by Skillman et al (2013), featuring a run-time simulated magnetic field injected by past AGN activity (color map). Bottom right panel: close up view of a radio relic in a galaxy clusters simulated using Eulerian AMR with run-time magnetic fields (assumed of primordial origin) by Wittor et al (2017), and imposing that only quasi-perpendicolar shocks efficiently accelerate electrons. The zoom covers $\sim 3.2 \times 3.2 \mathrm{Mpc}^{2}$, the color map shows the projected gas temperature, the red contours the detectable radio emission at $1.4 \mathrm{GHz}$, and the vectors show the local direction of magnetic fields.

halos (Pfrommer et al 2008, see also Van Weeren et al, this book). Injected CR protons were also reported to significantly reduce the star formation efficiency in small galaxies, as well as to affect the total mass-to-light ratio of small halos at the faint-end of the luminosity distribution (Jubelgas et al, 2008). Cosmic rays injected in galaxy clusters should indeed lower the effective adiabatic index of the gas $\Gamma_{\text {eff }} \leq \Gamma=5 / 3$, and within cool core regions the pressure from cosmic rays was found to approach equipartition with the thermal pressure, thereby enhancing the gas pressure and density and affecting the SZ signal (Pfrommer et al, 2007). In general, the most resolved SPH simulations reported on average $\sim 5 \%$ CRs to thermal gas energy ratio within $R_{\text {vir }}$ of clusters simulated with a non-radiative setup, and $\sim 10-30 \%$ with the inclu- 

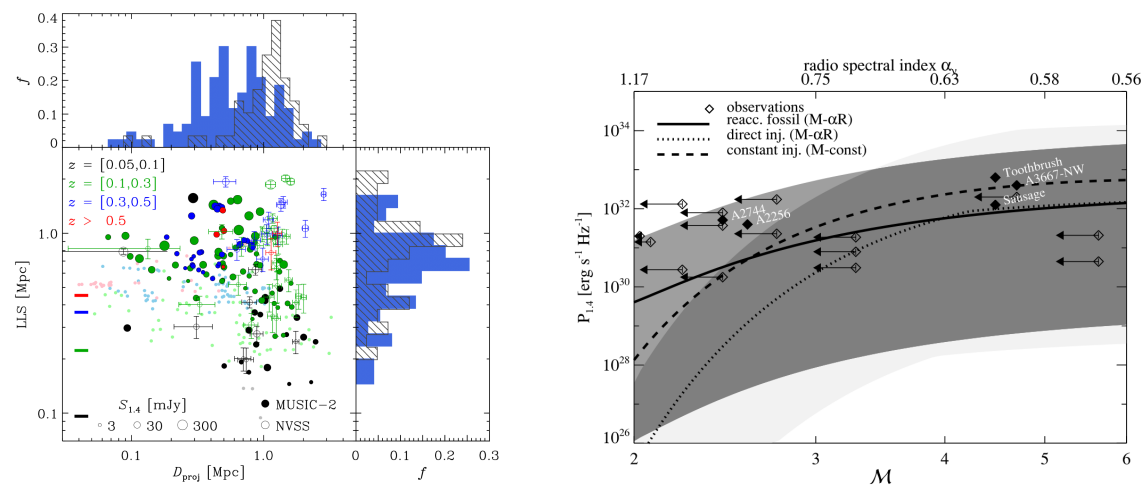

Fig. 9 Left panel: comparison between the projected distance and the largest linear scale (LLS) of observed (filled circles) and simulated (open circles with different colors for different redshift) radio relics, from Nuza et al (2017). Right panel: relation between the relic radio power and the shock Mach number for observed relics (diamonds, with uncertainties given by the length of horizontal arrows) and reference acceleration models (with thickness of the areas given by the typical range of uncertainties in the shock thickness, magnetic field, and downstream temperature and density) for three models: a model with fixed acceleration efficiency ("constant inj."), a model based on the DSA acceleration of fresh electrons only ("direct inj.") and a model including shock re-accelerated electrons ("reacc.fossil"). Adapted from (Pinzke et al 2013).

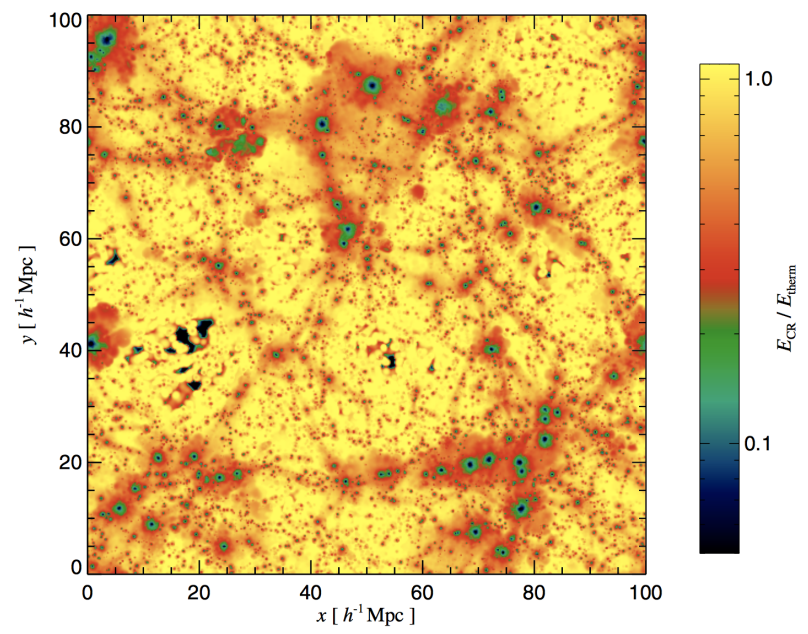

Fig. 10 Projected maps of the pressure ratio between cosmic ray protons and thermal gas, in an SPH cosmological simulations by Jubelgas et al 2008.

sion of radiative gas cooling, with little dependence on the cluster dynamical state (Jubelgas et al, 2008). At the epoch in which such simulations were performed, their predictions resulted to be below the available Fermi LAT upper limits, referred to $\sim 2.5$ years since the start of the mission (Fig 12 ). 

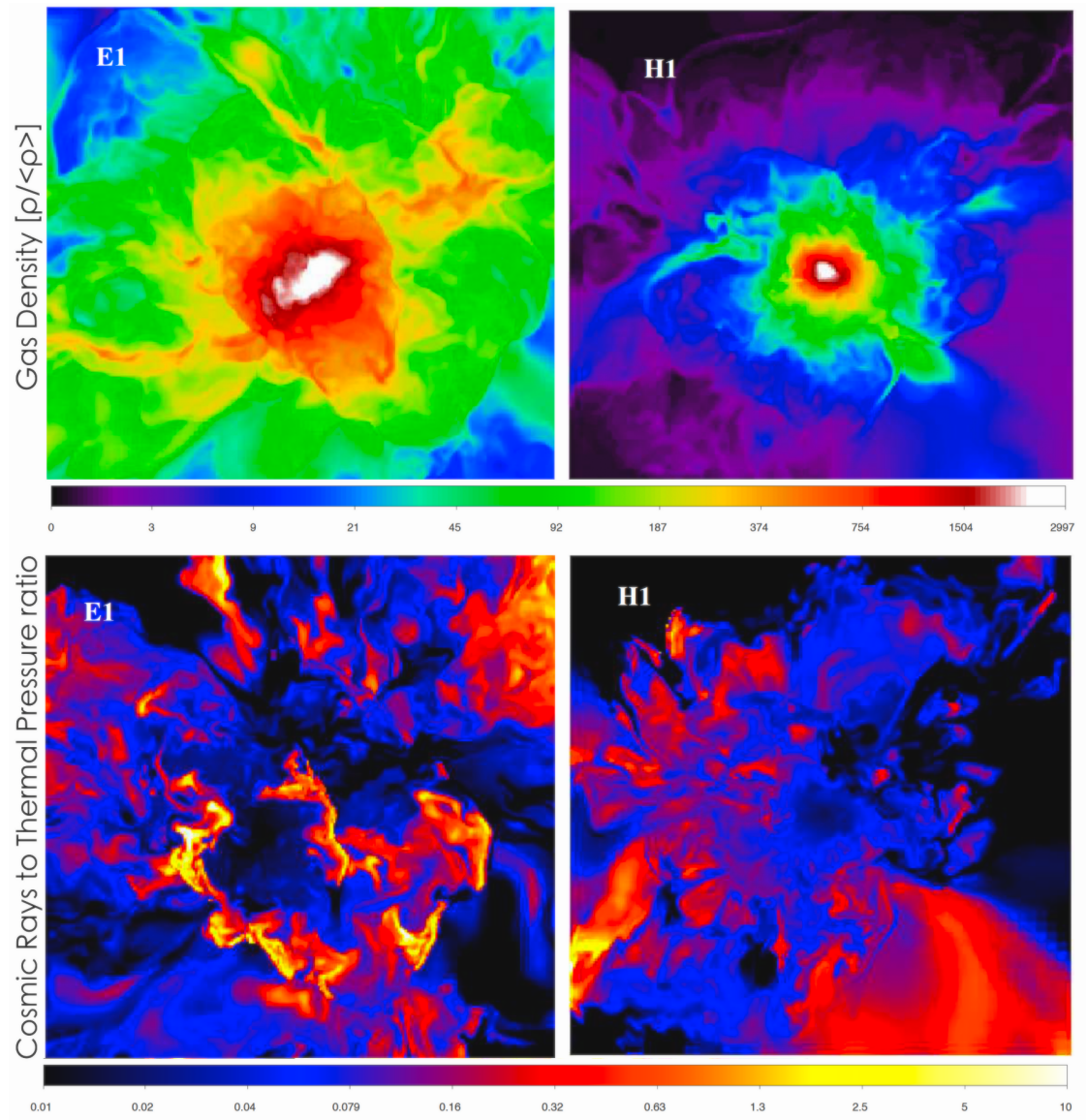

Fig. 11 Gas density map (top) and pressure ratio between cosmic ray protons and thermal gas (bottom) for thin slices through the center of two galaxy clusters simulated with the ENZO grid code by Vazza et al 2012a).

Later on, cosmological grid simulations (Fig 11) reached the necessary spatial detail to model the injection and advection of CRs with a two-fluid approach as well (Vazza et al, 2012a, 2014), testing more modern versions of diffusive shock acceleration, and also including non-gravitational gas physics and shock re-acceleration physics (Vazza et al, 2013, 2016). A few key differences were found respect to SPH results, ascribed to the different way in which the advection and entropy mixing of the two methods are numerically handled (e.g. Agertz et al, 2007, Vazza et al, 2011), as well to the fact that these more recent simulations adopted revised version of the diffusive shock (re)acceleration efficiency by Kang and Jones (2007) and Kang and Ryu (2013). As most of these works were performed after the report of the latest Fermi LAT upper limits (Ackermann et al, 2014), they could directly address the issue of limiting the acceleration efficiency of CR protons from the comparison with observations, as also most of the early grid simulations predicted hadronic fluxes in 


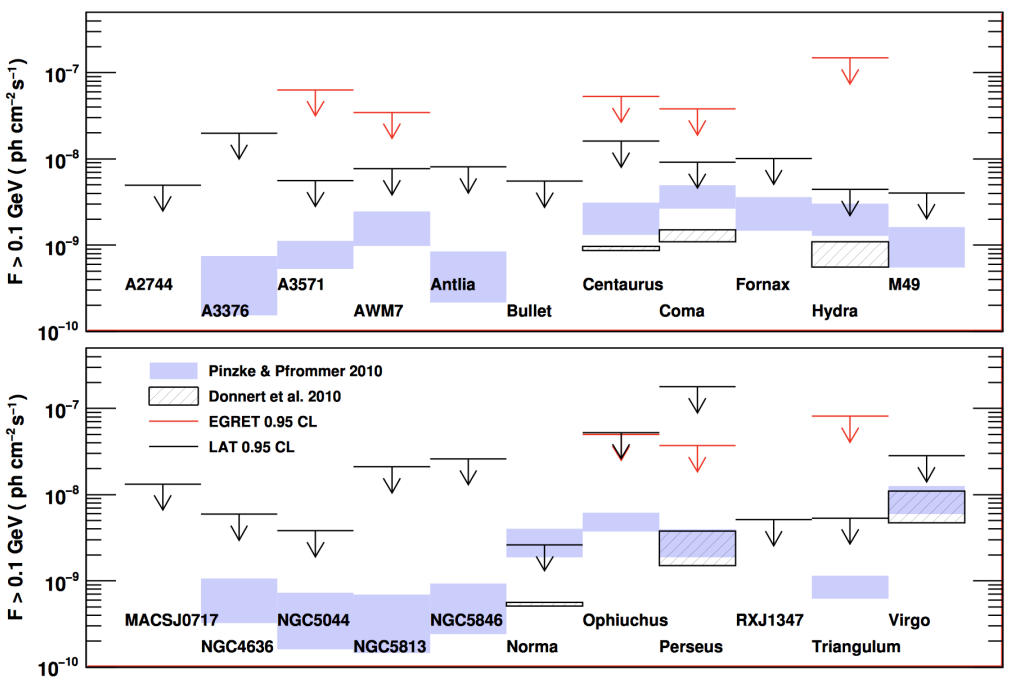

Fig. 12 Photon flux upper limits derived from Fermi-LAT by Ackermann et al (2010) for the observations of several galaxy clusters (assuming unresolved gamma-ray emission) are compared to EGRET results (Reimer et al 2003). The rectangles report the predictions based on SPH simulations using a two-fluid model (Pinzke and Pfrommer 2010) and SPH simulations using a semi-analytical modeling of cosmic ray protons and radio emission (Donnert et al 2010). Taken from Ackermann et al 2010).

excess to observations (e.g. Vazza et al, 2013).

In most tested models, the predicted hadronic $\gamma$-ray emission is in excess to Fermi LAT limits, for a $\sim 30-60 \%$ of simulated clusters (Vazza et al, 2016). The inclusion of cooling and feedback was found to significantly worsen the problem ${ }^{2}$ At variance with previous SPH works, Eulerian simulations reported a larger variance of the CR to gas pressure within the virial volume of clusters with the same mass, following from an overall stronger dependence of clusters' dynamical history and the injection and re-acceleration of CRs. If this is the case, the $\sim 1 \%$ upper limits on the ratio between CR energy and thermal gas energy that can be derived from Fermi LAT should mostly constrain galaxy clusters with a highly disturbed dynamical state, while in the rest of more relaxed systems the energy ratio within the virial volume may be a factor $\sim 10$ smaller, i.e. $\sim 0.1 \%$ (Vazza et al, 2016). As an example, the left panel of Fig 14 shows the predicted $\gamma$-ray emission level for clusters simulated in Vazza et al (2016) and contrasts it with the Fermi LAT limits (Ackermann et al, 2014). In the baseline scenario the authors tested the (re-)acceleration efficiencies derived by Kang and Jones (2007) or Kang and Ryu (2013), which are based on the outcome of 1-dimensional diffusion-convection equation of cosmic shocks, and allows to study the effect of simplistic acceleration efficiency that simply scales with the shock Mach

2 We notice that more recent works also included the effects of CR diffusion (Salem et al 2016, Jacob et al 2018), and CR streaming (Wiener et al 2017) in affecting the launching of star formation wind and their impact on the surrounding medium, even if the role of this in the total energy budget of CR in the intracluster medium on $\sim$ Mpc scales is not significant. 
number. Among the tested models, only a model in which the shock (re)acceleration efficiency of CR protons is bound to the low efficiency floor of $\eta=0.1 \%$ (in the very simplistic assumption that the acceleration efficiency is independent on the shock Mach number) would produce $\gamma$-ray emission below Fermi LAT limits. This corresponds to a very flat profile for the pressure ratio between accelerated CRs and thermal gas in most galaxy clusters at $z=0$, giving $\leq 0.5 \%$ within $R_{200}$ of most objects. However, such a small acceleration efficiency for CR protons poses in several cases problems to the "simple" explanation of radio relics with DSA (see below).

A crucial problem of every cosmological simulation is that the CR acceleration efficiency, $\eta(\mathscr{M})=f_{\mathrm{CR}} / f_{\text {diss }}$, defined as the ratio between the injected CR energy flux and the total kinetic energy dissipated by shocks, is unknown and must be guessed based on other works. The present state of affairs is summarized in Figure 13, which shows several relevant examples of acceleration efficiency functions considered by a few cosmological simulations from the last decade. The KJ 2007 model is based on 1-dimensional diffusion-convection simulations of diffusive shock acceleration by Kang and Jones (2007), while the KR2013 model is an updated version of the same baseline mode, including the effect of energy dissipation by Alfven waves amplified at the shock, yielding a lower efficiency (Kang and Ryu, 2013). Both models were included in Eulerian simulations (Vazza et al, 2012a, 2016), also including the effect of reaccelerated cosmic rays. The EN+2007 model is similar to the KJ 2007 model, but assumes a fixed acceleration efficiency $(0.3$ or 0.03$)$ as a function of the gas temperature ( $\mathrm{T}=1 \mathrm{keV}$ or $\mathrm{T}=0.1 \mathrm{keV}$ in the Figure, respectively). This acceleration model was used in SPH simulations (Pfrommer et al, 2007, 2008; Pinzke and Pfrommer 2010).

Finally, the KR2013+CS2014 hybrid model is an updated version of the Kang and Ryu (2013) model, which includes the effect of a distribution of shock obliquities at cosmic shocks, as discussed in Eulerian grid simulations in Vazza et al (2016) and tested in detail by Wittor et al (2017). If the probability distribution of shock obliquities is random $(P(\theta) \propto \sin (\theta))$, only $\approx 0.3$ of shocks have $\theta \leq 45^{\circ}$ (i.e. are quasi-parallel). In this range of shocks, Caprioli and Spitkovsky (2014) have reported that the shock acceleration efficiency is $\approx 0.5$ of the efficiency in Kang and Ryu (2013) for the same Mach number range. Therefore, the combination of these two factors should roughly mimic the reduced acceleration efficiency of CR protons in quasi-perpendicular shocks in large-scale structures (see also discussion below).

Clearly, the differences among models are large, within a factor $\sim 10$ for strong shocks $(\mathscr{M} \geq 6)$, but (more worryingly) of order $\geq 100$ in the regime which is more crucial to cluster merger shocks, $\mathscr{M} \leq 4$. Most of CRs injected in structure formation shocks should come from these shocks, as well as most of the observed radio relic emission (e.g. Van Weeren et al., this topical collection), and thus any small difference in the frequency of shocks in this regime, as well as on the assumed acceleration efficiency, will have a dramatic impact in the predicted budget of CRs. However, it seems now clear that deeper changes in the simplistic view of CR proton acceleration in the ICM must be introduced, in order to cope with observational evidences. 


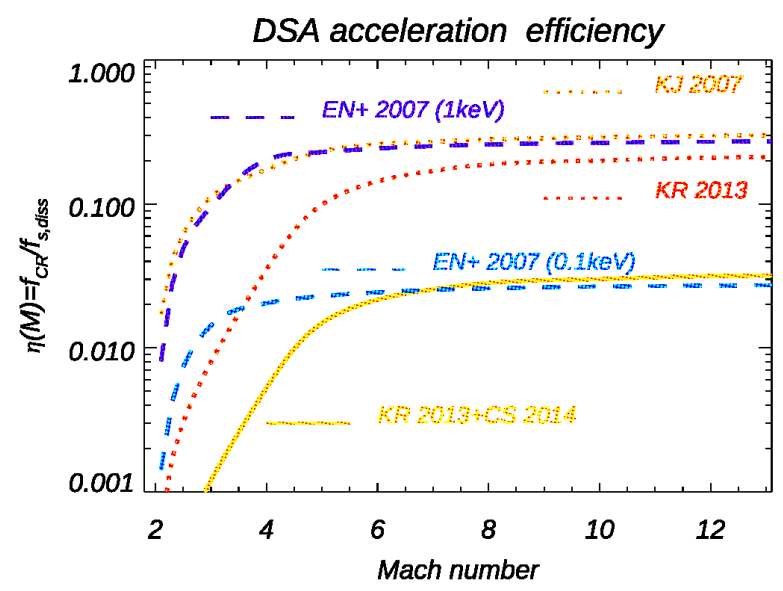

Fig. 13 Different proposed acceleration efficiencies function $\eta(\mathscr{M})$ as a function of shock Mach number, where $\eta(\mathscr{M})=f_{\mathrm{CR}} / f_{\text {diss }}$ is the ratio between the injected cosmic ray energy flux and the total kinetic energy dissipated by shocks. The various models in different colors are: KJ2007 for Kang and Jones (2007), KR2013 for Kang and Ryu (2013), EN+2007 for Enßlin et al (2007) (including two different gas temperatures) and KR 2013+CS 2014 for an "hybrid model" derived by combining the results of Kang and Ryu (2013) and the obliquity-dependent results from Caprioli and Spitkovsky (2014), as in Vazza et al (2016).

\subsection{Possible solutions to the lack of cosmic ray protons dilemma}

Based on the historic trend of findings in cosmological simulations and after the report of Fermi LAT non-detections (combined with the observation of radio emission from relativistic electrons in many of the same objects) several possible explanations have been explored and tested, all with still inconclusive solutions.

A first possibility is to relax the standard assumption that the overall distribution of CR protons remains confined within galaxy clusters for longer than a Hubble time, due to their trapping into the tangled intracluster magnetic field (Völk et al, 1996, Berezinsky et al, 1997). A few works have indeed raised the possibility that CR protons may stream out of the innermost cluster regions, at a velocity $\geq v_{a}$ (where $v_{a}$ is the local Alfven velocity) on large scales, which would significantly lower the expected hadronic emission as the signal drops as $\propto n^{2}$, thereby transforming peaked CR profiles into asymptotically flat ones. This possibility has been first proposed by Enßlin et al (2011) in the case of galaxy clusters, and has been later addressed with more sophisticated methods by other authors (Wiener et al, 2013; Zandanel et al 2014, Pinzke et al, 2017). At present, the jury is still out on the feasibility of such mechanism in the plasma conditions of the ICM, due to the complex and time-dependent interplay between plasma turbulence, MHD waves and CRs, that is expected to be present in every galaxy cluster (Wiener et al, 2018). Although theoretically challenging to justify, the $\geq v_{a}$ streaming of CRs would in principle alleviate the tension with Fermi LAT limits and explain the lack of radio emission in radio quiet 
clusters. However, this solution cannot account for the lack of hadronic emission in clusters where instead large-scale radio halo emission is observed (see Van Weeren et al., this topical collection), because at least there CR protons should be present if the emission must be explained away by a secondary electron model. This makes it overall unlikely that cosmic ray streaming (at least, alone) can explain the lack of detections of hadronic emission reported by Fermi LAT.

An other class of possible solutions focuses on the revision of the assumed particle acceleration efficiency of CR protons. Vazza and Brüggen (2014) and Vazza et al (2015) made the case of observed "double" radio relics (see Van Weeren et al., this topical collection) which also expose open problems of the "standard" DSA acceleration model applied to the ICM, in the sense that if observed double radio relics were uniquely the product of DSA acceleration, a significant amount of cosmic ray protons should be present there too. From the modelling of observed radio relic emission it is indeed possible to guess the shock parameters (including the shock Mach number), as well as the total volume swept in the past by relics; from the combination of these parameters it is possible to predict the total amount of CR protons that must have been injected (and confined) in the ICM, for different formulations of the DSA scenario. When these estimates are applied to real objects, in several cases the implied injection ratio between electron and proton is unexpectedly high $(\geq 0.1-1)$ (Vazza and Brüggen, 2014; Brunetti and Jones, 2014). In other words, the simultaneous radio observation of relativistic electrons and the non detection of CR protons via $\gamma$-rays is explained only if the acceleration from merger shock tend to privilege (or equally accelerate) electrons and protons, which is at variance with expected in the DSA scenario (where the expected efficiency should be $\sim 10^{-5}-10^{-3}$ for typical merger shocks).

A solution capable of alleviating part of the problem with radio relics is that part (or most) of the emission in relics comes from shock re-accelerated electrons, meaning that a pool of "fossil" electrons with a significant energy (albeit not radio emitting) is often present in the volume swept-up by merger shocks (e.g. Markevitch et al 2005; Pinzke et al, 2013). In this case, the conversion efficiency of shock kinetic energy and radio power gets boosted (e.g. Kang et al, 2012a; Pinzke et al, 2013) and even relatively weak shocks $(\mathscr{M} \sim 1.3-2.5)$ can produce detectable radio emission. In this scenario, while the CR proton acceleration from merger shocks in the ICM can be considered low enough to never produce a significant level of hadronic emission, the acceleration of CR electrons gets boosted by the presence of a "fossil" population of electrons. Therefore, a key prerequisite for this mechanism to work is the presence of volume filling CR electrons mixed with the thermal ICM. A large number of radio galaxies (active nowadays or in the past) is surely present in the ICM, and a number of observations reported convincing evidence for a close association between diffuse radio emission an localized sources of seed electrons (e.g. Van Weeren, this topical collection). Moreover, Pinzke et al (2013) have shown that indeed a bath of relic electrons can be present in the outer regions of clusters, with very long cooling times $\left(\geq t_{H}\right)$, as a result of shock acceleration in cluster outskirts. While this idea can address the presence of relic emission also in shocks with a weak Mach number, in 
order for this scenario to cope with the lack of hadronic emission even in systems with prominent relic emission (suggesting intense shock crossing in the recent past) fossil protons must be absent instead. This may put some tension on the possibility in which fossil cosmic ray electrons come from previous shocks (which would have injected cosmic ray protons as well), and it also requires that the injection of CRs from supernovae and AGN to be overall dominated by cosmic ray electrons, which is non trivial to achieve.

A third possible solution is the incorporation of key "microscopic" details of the shock electron and proton acceleration, which have been overlooked in the past, at least for problems relevant to galaxy clusters. While it is still impossible to fully include all physical scales which are key to resolve in the simulation of shock acceleration (see Sections above in this chapter), recent cosmological simulations also including magnetic fields have attempted to incorporate recent studies from Particle In Cell (PIC) simulations, (Caprioli and Spitkovsky, 2014, Guo et al, 2014a), in which the shock obliquity $(\theta)$ is a key parameter to regulate the acceleration of, both, electrons and protons by cosmic shocks.

In the Earth's bow shock and in interplanetary shocks, electrons are directly observed to be accelerated to relativistic energies preferentially in quasi-perpendicular configurations, i.e. $\theta>40-50^{\circ}$ (e.g. Shimada et al, 1999, Oka et al, 2006). Recent PIC simulations of electron acceleration by weak intracluster shocks observed that only quasi-perpendicular shocks can pre-accelerate electrons, via shock-driftacceleration (SDA), before being injected into DSA (Guo et al, 2014c a). With highly spatially and temporally resolved simulations of forming clusters, Wittor et al (2016) and Wittor et al (2017) have tested the above ideas, making the simplifying approximation that the acceleration of CR electrons is entirely "switched off" for quasiparallel shocks $\left(\theta \leq 50^{\circ}\right)$, while conversely the acceleration of CR protons is entirely "switched off" for quasi-perpendicular shocks $\left(\theta \leq 50^{\circ}\right)$, based on the results of Guo et al (2014c) and Caprioli and Spitkovsky (2014). This introduces an angle-dependent acceleration efficiency in the cosmological run, $\eta(\mathscr{M}, \theta)$, which gets very small for cosmic ray protons during merger shocks at low redshift, because the ICM magnetic fields is tangled for $\leq$ Mpc scales (see e.g. Donnert et al. this topical collection) and the distribution of shock obliquities gets close to random, thus in this regime the majority of shocks is quasi-perpendicular. As a consequence of this, the energy ratio between CR protons and thermal energy is reduced by a factor $\sim 50 \%$ if only quasiparallel shocks are concerned, and the predicted hadronic $\gamma$-ray emission within $R_{200}$ gets reduced to $\sim 25-30 \%$ of more standard estimate (see right panel of Fig 14). This effect is in general not large enough to fully alleviate the tension with Fermi LAT non detections, yet it suggests a new level of complexity and realism to the future testing of DSA models.

As already commented in Sec. 3, Kang and Ryu (2018) reported that for quasiparallel shocks and ICM conditions $(\mathscr{M} \sim 2-3)$, when postshock waves are isotropized by plasma processes, the Alfvénic drift could reduce the injection efficiency of CRs by a factor $\sim 5$ with respect to more standard estimates.

In summary, from the stringent comparison with Fermi LAT non detections of hadronic $\gamma$-ray emission, cosmological simulations have established that only a neg- 

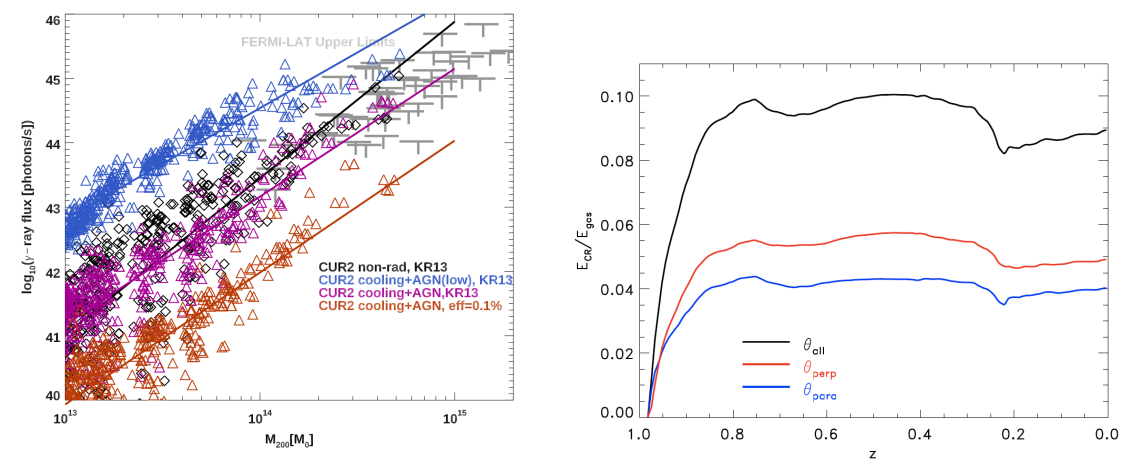

Fig. 14 Left panel: hadronic emission for ENZO -simulated clusters in Vazza et al (2016) in the 0.2-200 $\mathrm{GeV}$ energy range. The gray symbols are the upper limits from the Fermi LAT catalog in the same energy range (Ackermann et al 2014). The different colors refer to resimulations of the same set of galaxy clusters, with variations of the assumed gas physics (i.e. non-radiative vs radiative gas physics, AGN feedback) and of the assume cosmic ray acceleration efficiency ("KR13"=acceleration model assumed from Kang and Ryu 2013, "eff $=0.1 \%$ " assumes a fixed $10^{-3}$ acceleration efficiency of cosmic ray protons regardless of the shock Mach number. Taken from Vazza et al 2016). Right panel: evolution of the ration between the $\mathrm{CR}$ energy and the thermal gas energy for particles accreted onto a $\sim 10^{15} M_{\odot}$ simulated cluster in Wittor et al (2017), for the DSA efficiency model by Kang and Ryu 2013. or by further limiting the acceleration to quasi-parallel $\left(\theta_{\text {para }}\right)$ or quasi-perpendicular $\left(\theta_{\text {perp }}\right)$ shocks.

ligible energy in relativistic CR protons can be present in the intracluster medium (i.e. $\leq 1 \%$ of the total gas energy within the virial volume), for typical spectra of accelerated CRs with a power law index $\sim 2.1-2.3$, resulting from the combination of accretion and merger shocks. This poses challenges possibly to both our understanding of the injection of CR protons across cosmic environment, as well as of the advection and diffusion of CRs during clusters'evolution. However, the non-thermal components with the steep spectra of the power law indexes $\gtrsim 3$ can be produced by an ensemble of long-wavelength plasma motions with weak shocks as it was discussed in section 4.1. The soft components could contribute more into the nonthermal pressure in the intracluster medium. Such soft component is observed in the interplanetary medium (Fisk, 2015). Since the nonthermal component is dominated by sub-relativistic ions the sensitive high resolution X-ray spectroscopy may help to constrain it. The departures from the Maxwellian momentum distribution of the $\mathrm{Fe}$ ions at the cluster shocks can be potentially constrained with the next generation large effective area spectroscopy instruments aboard the ATHENA mission (see e.g. Barret et al 2018) and the X-Ray Microcalorimeter-Imaging Spectrometer which is planned for the Lynx observatory.

While it is preliminary to say if any of the above proposed solutions (or a combination of them) will be able to explain the missing hadronic emission from the ICM, it seems clear that only by jointly testing the "microscopic" view of particle acceleration at shocks and of the "macroscopic" view enabled by cosmological simulations of forming structures it will be possible to quantitatively test solutions to the challenges posed by radio and $\gamma$-ray observations. 
Acknowledgements A.M.B. thanks the staff of ISSI for their generous hospitality and assistance. The authors thank the referees for the constructive comments. A.M. Bykov and J.A. Kropotina were supported by the RSF grant 16-12-10225. Some of the modeling was performed at the "Tornado" subsystem of the St. Petersburg Polytechnic University supercomputing center and at the JSCC RAS. F.V. and acknowledges financial support from the European Union's Horizon 2020 program under the ERC Starting Grant "MAGCOW", no. 714196.

\section{References}

Ackermann M, Ajello M, Allafort A, Baldini L, Ballet J, Barbiellini G, Bastieri D, Bechtol K, Bellazzini R, Blandford RD, Blasi P, Bloom ED, Bonamente E, Borgland AW, Bouvier A, Brandt TJ, Bregeon J, Brigida M, Bruel P, Buehler R, Buson S, Caliandro GA, Cameron RA, Caraveo PA, Carrigan S, Casandjian JM, Cavazzuti E, Cecchi C, Çelik Ö, Charles E, Chekhtman A, Cheung CC, Chiang J, Ciprini S, Claus R, Cohen-Tanugi J, Colafrancesco S, Cominsky LR, Conrad J, Dermer CD, de Palma F, Silva EdCe, Drell PS, Dubois R, Dumora D, Edmonds Y, Farnier C, Favuzzi C, Frailis M, Fukazawa Y, Funk S, Fusco P, Gargano F, Gasparrini D, Gehrels N, Germani S, Giglietto N, Giordano F, Giroletti M, Glanzman T, Godfrey G, Grenier IA, Grondin MH, Guiriec S, Hadasch D, Harding AK, Hayashida M, Hays E, Horan D, Hughes RE, Jeltema TE, Jóhannesson G, Johnson AS, Johnson TJ, Johnson WN, Kamae T, Katagiri H, Kataoka J, Kerr M, Knödlseder J, Kuss M, Lande J, Latronico L, Lee SH, Lemoine-Goumard M, Longo F, Loparco F, Lott B, Lovellette MN, Lubrano P, Madejski GM, Makeev A, Mazziotta MN, Michelson PF, Mitthumsiri W, Mizuno T, Moiseev AA, Monte C, Monzani ME, Morselli A, Moskalenko IV, Murgia S, Naumann-Godo M, Nolan PL, Norris JP, Nuss E, Ohsugi T, Omodei N, Orlando E, Ormes JF, Ozaki M, Paneque D, Panetta JH, Pepe M, Pesce-Rollins M, Petrosian V, Pfrommer C, Piron F, Porter TA, Profumo S, Rainò S, Rando R, Razzano M, Reimer A, Reimer O, Reposeur T, Ripken J, Ritz S, Rodriguez AY, Romani RW, Roth M, Sadrozinski HFW, Sander A, Saz Parkinson PM, Scargle JD, Sgrò C, Siskind EJ, Smith PD, Spandre G, Spinelli P, Starck JL, Stawarz Ł, Strickman MS, Strong AW, Suson DJ, Tajima H, Takahashi H, Takahashi T, Tanaka T, Thayer JB, Thayer JG, Tibaldo L, Tibolla O, Torres DF, Tosti G, Tramacere A, Uchiyama Y, Usher TL, Vandenbroucke J, Vasileiou V, Vilchez N, Vitale V, Waite AP, Wang P, Winer BL, Wood KS, Yang Z, Ylinen T, Ziegler M (2010) GeV Gamma-ray Flux Upper Limits from Clusters of Galaxies. ApJL 717:L71-L78, DOI 10.1088/2041-8205/717/1/L71, 1006.0748

Ackermann M, Ajello M, Allafort A, Baldini L, Ballet J, Barbiellini G, Bastieri D, Belfiore A, Bellazzini R, Berenji B, Blandford RD, Bloom ED, Bonamente E, Borgland AW, Bottacini E, Brigida M, Bruel P, Buehler R, Buson S, Caliandro GA, Cameron RA, Caraveo PA, Casandjian JM, Cecchi C, Chekhtman A, Cheung CC, Chiang J, Ciprini S, Claus R, Cohen-Tanugi J, de Angelis A, de Palma F, Dermer CD, do Couto e Silva E, Drell PS, Dumora D, Favuzzi C, Fegan SJ, Focke WB, Fortin P, Fukazawa Y, Fusco P, Gargano F, Germani S, Giglietto N, Giordano F, Giroletti M, Glanzman T, Godfrey G, Grenier IA, Guillemot L, Guiriec S, Hadasch D, Hanabata Y, Okumura A, Orlando E, Ormes JF, Ozaki M, Paneque D, Parent D, Pesce-Rollins M, Pierbattista M, Piron F, Pohl M, Prokhorov D, Rainò 
S, Rando R, Razzano M, Reposeur T, Ritz S, Parkinson PMS, Sgrò C, Siskind EJ, Smith PD, Spinelli P, Strong AW, Takahashi H, Tanaka T, Thayer JG, Thayer JB, Thompson DJ, Tibaldo L, Torres DF, Tosti G, Tramacere A, Troja E, Uchiyama Y, Vandenbroucke J, Vasileiou V, Vianello G, Vitale V, Waite AP, Wang P, Winer BL, Wood KS, Yang Z, Zimmer S, Bontemps S (2011) A Cocoon of Freshly Accelerated Cosmic Rays Detected by Fermi in the Cygnus Superbubble. Science 334:1103-, DOI 10.1126/science.1210311

Ackermann M, Ajello M, Albert A, Allafort A, Atwood WB, Baldini L, Ballet J, Barbiellini G, Bastieri D, Bechtol K, Bellazzini R, Bloom ED, Bonamente E, Bottacini E, Brandt TJ, Bregeon J, Brigida M, Bruel P, Buehler R, Buson S, Caliandro GA, Cameron RA, Caraveo PA, Cavazzuti E, Chaves RCG, Chiang J, Chiaro G, Ciprini S, Claus R, Cohen-Tanugi J, Conrad J, D’Ammando F, de Angelis A, de Palma F, Dermer CD, Digel SW, Drell PS, Drlica-Wagner A, Favuzzi C, Franckowiak A, Funk S, Fusco P, Gargano F, Gasparrini D, Germani S, Giglietto N, Giordano F, Giroletti M, Godfrey G, Gomez-Vargas GA, Grenier IA, Guiriec S, Gustafsson M, Hadasch D, Hayashida M, Hewitt J, Hughes RE, Jeltema TE, Jóhannesson G, Johnson AS, Kamae T, Kataoka J, Knödlseder J, Kuss M, Lande J, Larsson S, Latronico L, Llena Garde M, Longo F, Loparco F, Lovellette MN, Lubrano P, Mayer M, Mazziotta MN, McEnery JE, Michelson PF, Mitthumsiri W, Mizuno T, Monzani ME, Morselli A, Moskalenko IV, Murgia S, Nemmen R, Nuss E, Ohsugi T, Orienti M, Orlando E, Ormes JF, Perkins JS, Pesce-Rollins M, Piron F, Pivato G, Rainò S, Rando R, Razzano M, Razzaque S, Reimer A, Reimer O, Ruan J, Sánchez-Conde M, Schulz A, Sgrò C, Siskind EJ, Spandre G, Spinelli P, Storm E, Strong AW, Suson DJ, Takahashi H, Thayer JG, Thayer JB, Thompson DJ, Tibaldo L, Tinivella M, Torres DF, Troja E, Uchiyama Y, Usher TL, Vandenbroucke J, Vianello G, Vitale V, Winer BL, Wood KS, Zimmer S, Fermi-LAT Collaboration, Pinzke A, Pfrommer C (2014) Search for Cosmic-Ray-induced Gamma-Ray Emission in Galaxy Clusters. ApJ 787:18, DOI 10.1088/0004-637X/787/1/18, 1308.5654

Ackermann M, Ajello M, Albert A, Atwood WB, Baldini L, Ballet J, Barbiellini G, Bastieri D, Bechtol K, Bellazzini R, Bissaldi E, Blandford RD, Bloom ED, Bonino R, Bottacini E, Bregeon J, Bruel P, Buehler R, Caliandro GA, Cameron RA, Caragiulo M, Caraveo PA, Casandjian JM, Cavazzuti E, Cecchi C, Charles E, Chekhtman A, Chiaro G, Ciprini S, Cohen-Tanugi J, Conrad J, Cutini S, D’Ammando F, de Angelis A, de Palma F, Desiante R, Digel SW, Di Venere L, Drell PS, Favuzzi C, Fegan SJ, Fukazawa Y, Funk S, Fusco P, Gargano F, Gasparrini D, Giglietto N, Giordano F, Giroletti M, Godfrey G, Green D, Grenier IA, Guiriec S, Hays E, Hewitt JW, Horan D, Jóhannesson G, Kuss M, Larsson S, Latronico L, Li J, Li L, Longo F, Loparco F, Lovellette MN, Lubrano P, Madejski GM, Maldera S, Manfreda A, Mayer M, Mazziotta MN, Michelson PF, Mitthumsiri W, Mizuno T, Monzani ME, Morselli A, Moskalenko IV, Murgia S, Nuss E, Ohsugi T, Orienti M, Orlando E, Ormes JF, Paneque D, Pesce-Rollins M, Petrosian V, Piron F, Pivato G, Porter TA, Rainò S, Rando R, Razzano M, Reimer A, Reimer O, Sánchez-Conde M, Sgrò C, Siskind EJ, Spada F, Spandre G, Spinelli P, Tajima H, Takahashi H, Thayer JB, Tibaldo L, Torres DF, Tosti G, Troja E, Vianello G, Wood KS, Zimmer S, The Fermi-LAT Collaboration, Rephaeli Y (2016) Search for Gamma-Ray Emission from the Coma Cluster with Six Years of Fermi-LAT Data. ApJ 819:149, 
DOI 10.3847/0004-637X/819/2/149, 1507.08995

Agertz O, Moore B, Stadel J, Potter D, Miniati F, Read J, Mayer L, Gawryszczak A, Kravtsov A, Nordlund Å, Pearce F, Quilis V, Rudd D, Springel V, Stone J, Tasker E, Teyssier R, Wadsley J, Walder R (2007) Fundamental differences between SPH and grid methods. MNRAS 380:963-978, DOI 10.1111/j.1365-2966.2007.12183. $\mathrm{x}$, arXiv: astro-ph/0610051

Antecki T, Schlickeiser R, Zhang M (2013) Stochastic Acceleration of Suprathermal Particles under Pressure Balance Conditions. ApJ 764:89, DOI 10.1088/ 0004-637X/764/1/89

Araya-Melo PA, Aragón-Calvo MA, Brüggen M, Hoeft M (2012) Radio emission in the cosmic web. MNRAS 423:2325-2341, DOI 10.1111/j.1365-2966.2012.21042. $\mathrm{x}, 1204.1759$

Artemyev AV, Neishtadt AI, Vainchtein DL, Vasiliev AA, Vasko IY, Zelenyi LM (2018) Trapping (capture) into resonance and scattering on resonance: Summary of results for space plasma systems. Communications in Nonlinear Science and Numerical Simulations 65:111-160, DOI 10.1016/j.cnsns.2018.05.004

Barret D, Lam Trong T, den Herder JW, Piro L, Cappi M, Houvelin J, Kelley R, Mas-Hesse JM, Mitsuda K, Paltani S, Rauw G, Rozanska A, Wilms J, Bandler S, Barbera M, Barcons X, Bozzo E, Ceballos MT, Charles I, Costantini E, Decourchelle A, den Hartog R, Duband L, Duval JM, Fiore F, Gatti F, Goldwurm A, Jackson B, Jonker P, Kilbourne C, Macculi C, Mendez M, Molendi S, Orleanski P, Pajot F, Pointecouteau E, Porter F, Pratt GW, Prêle D, Ravera L, Sato K, Schaye J, Shinozaki K, Thibert T, Valenziano L, Valette V, Vink J, Webb N, Wise M, Yamasaki N, Douchin F, Mesnager JM, Pontet B, Pradines A, Branduardi-Raymont G, Bulbul E, Dadina M, Ettori S, Finoguenov A, Fukazawa Y, Janiuk A, Kaastra J, Mazzotta P, Miller J, Miniutti G, Naze Y, Nicastro F, Scioritino S, Simonescu A, Torrejon JM, Frezouls B, Geoffray H, Peille P, Aicardi C, André J, Daniel C, Clénet A, Etcheverry C, Gloaguen E, Hervet G, Jolly A, Ledot A, Paillet I, Schmisser R, Vella B, Damery JC, Boyce K, Dipirro M, Lotti S, Schwander D, Smith S, Van Leeuwen BJ, van Weers H, Clerc N, Cobo B, Dauser T, Kirsch C, Cucchetti E, Eckart M, Ferrando P, Natalucci L (2018) The ATHENA X-ray Integral Field Unit (X-IFU). In: Society of Photo-Optical Instrumentation Engineers (SPIE) Conference Series, Society of Photo-Optical Instrumentation Engineers (SPIE) Conference Series, vol 10699, p 106991G, DOI 10.1117/12.2312409, 1807.06092

Battaglia N, Pfrommer C, Sievers JL, Bond JR, Enßlin TA (2009) Exploring the magnetized cosmic web through low-frequency radio emission. MNRAS 393:10731089, DOI 10.1111/j.1365-2966.2008.14136.x, 0806.3272

Berezinsky VS, Blasi P, Ptuskin VS (1997) Clusters of Galaxies as Storage Room for Cosmic Rays. ApJ 487:529-+, DOI 10.1086/304622, arXiv:astro-ph/ 9609048

Biffi V, Planelles S, Borgani S, Rasia E, Murante G, Fabjan D, Gaspari M (2018) The origin of ICM enrichment in the outskirts of present-day galaxy clusters from cosmological hydrodynamical simulations. MNRAS 476:2689-2703, DOI $10.1093 / \mathrm{mnras} / \mathrm{sty} 363,1801.05425$

Böhringer H, Werner N (2010) X-ray spectroscopy of galaxy clusters: studying astrophysical processes in the largest celestial laboratories. The Astronomy and As- 
trophysics Review 18:127-196, DOI 10.1007/s00159-009-0023-3

Bonafede A, Brüggen M, van Weeren R, Vazza F, Giovannini G, Ebeling H, Edge AC, Hoeft M, Klein U (2012) Discovery of radio haloes and double relics in distant MACS galaxy clusters: clues to the efficiency of particle acceleration. MNRAS 426:40-56, DOI 10.1111/j.1365-2966.2012.21570.x, 1206.6102

Brown S, Emerick A, Rudnick L, Brunetti G (2011) Probing the Off-state of Cluster Giant Radio Halos. ApJL 740:L28+, DOI 10.1088/2041-8205/740/1/L28, 1109. 0316

Brüggen M, Bykov A, Ryu D, Röttgering H (2012) Magnetic Fields, Relativistic Particles, and Shock Waves in Cluster Outskirts. Space Science Reviews 166:187213, DOI 10.1007/s11214-011-9785-9, 1107.5223

Brunetti G (2017) Gamma rays from galaxy clusters. In: 6th International Symposium on High Energy Gamma-Ray Astronomy, American Institute of Physics Conference Series, vol 1792, p 020009, DOI 10.1063/1.4968894

Brunetti G, Jones TW (2014) Cosmic Rays in Galaxy Clusters and Their Nonthermal Emission. International Journal of Modern Physics D 23:1430007-98, DOI 10.1142/S0218271814300079, 1401.7519

Brunetti G, Lazarian A (2007) Compressible turbulence in galaxy clusters: physics and stochastic particle re-acceleration. MNRAS 378:245-275, DOI 10.1111/j. 1365-2966.2007.11771.x, astro-ph/0703591

Brunetti G, Lazarian A (2011a) Acceleration of primary and secondary particles in galaxy clusters by compressible MHD turbulence: from radio haloes to gamma-rays. MNRAS 410:127-142, DOI 10.1111/j.1365-2966.2010.17457.x, 1008.0184

Brunetti G, Lazarian A (2011b) Particle reacceleration by compressible turbulence in galaxy clusters: effects of a reduced mean free path. MNRAS 412:817-824, DOI 10.1111/j.1365-2966.2010.17937.x, 1011.1198

Brunetti G, Venturi T, Dallacasa D, Cassano R, Dolag K, Giacintucci S, Setti G (2007a) ApJL 670:L5-L8, DOI 10.1086/524037, arXiv:0710.0801

Brunetti G, Venturi T, Dallacasa D, Cassano R, Dolag K, Giacintucci S, Setti G (2007b) Cosmic Rays and Radio Halos in Galaxy Clusters: New Constraints from Radio Observations. ApJL 670:L5-L8, DOI 10.1086/524037,0710.0801

Brunetti G, Blasi P, Reimer O, Rudnick L, Bonafede A, Brown S (2012) Probing the origin of giant radio haloes through radio and $\gamma$-ray data: the case of the Coma cluster. MNRAS 426:956-968, DOI 10.1111/j.1365-2966.2012.21785.x, 1207 . 3025

Brunetti G, Zimmer S, Zandanel F (2017) Relativistic protons in the Coma galaxy cluster: first gamma-ray constraints ever on turbulent reacceleration. MNRAS 472:1506-1525, DOI 10.1093/mnras/stx2092, 1707.02085

Bykov AM (2001) Particle Acceleration and Nonthermal Phenomena in Superbubbles. Space Science Reviews 99:317-326

Bykov AM (2014) Nonthermal particles and photons in starburst regions and superbubbles. The Astronomy and Astrophysics Review 22:77, DOI 10.1007/ s00159-014-0077-8

Bykov AM, Toptygin I (1993) Particle kinetics in highly turbulent plasmas (renormalization and self-consistent field methods). Physics Uspekhi 36:1020-1052, DOI 
10.1070/PU1993v036n11ABEH002179

Bykov AM, Toptygin IN (1983) Magnetohydrodynamic Turbulence in the Cosmic Rays Confinement Regions of Galaxy. International Cosmic Ray Conference 9:247

Bykov AM, Toptygin IN (1987) Effect of shocks on interstellar turbulence and cosmic-ray dynamics. Astrophys. Space Sci. 138:341-354, DOI 10.1007/ BF00637855

Bykov AM, Toptygin IN (1990) Theory of charged-particle acceleration by a collection of shock-waves in a turbulent medium. Sov Phys JETP 71:702-708

Bykov AM, Bloemen H, Uvarov YA (2000) Nonthermal emission from clusters of galaxies. A \& A 362:886-894

Bykov AM, Dolag K, Durret F (2008a) Cosmological Shock Waves. Space Science Reviews 134:119-140, DOI 10.1007/s11214-008-9312-9,0801.0995

Bykov AM, Paerels FBS, Petrosian V (2008b) Equilibration Processes in the WarmHot Intergalactic Medium. Space Science Reviews 134:141-153, DOI 10.1007/ s11214-008-9309-4, 0801.1008

Bykov AM, Ellison DC, Osipov SM, Vladimirov AE (2014) Magnetic Field Amplification in Nonlinear Diffusive Shock Acceleration Including Resonant and Nonresonant Cosmic-Ray Driven Instabilities. ApJ 789:137, DOI 10.1088/0004-637X/ 789/2/137, 1406.0084

Bykov AM, Ellison DC, Osipov SM (2017) Nonlinear Monte Carlo model of superdiffusive shock acceleration with magnetic field amplification. Physical Review E 95(3):033207, DOI 10.1103/PhysRevE.95.033207, 1703.01160

Bykov AM, Ellison DC, Marcowith A, Osipov SM (2018) Cosmic Ray Production in Supernovae. Space Science Reviews 214:41, DOI 10.1007/s11214-018-0479-4, 1801.08890

Caprioli D, Spitkovsky A (2014) Simulations of Ion Acceleration at Non-relativistic Shocks. I. Acceleration Efficiency. ApJ 783:91, DOI 10.1088/0004-637X/783/2/ $91,1310.2943$

Cavaliere A, Lapi A (2013) The astrophysics of the intracluster plasma. Physics Reports 533:69-94, DOI 10.1016/j.physrep.2013.08.001, 1308.6673

Chuzhoy L, Loeb A (2004) Element segregation in giant galaxies and X-ray clusters. MNRAS 349:L13-L17, DOI 10.1111/j.1365-2966.2004.07688.x, astro-ph/ 0312472

de Gasperin F, van Weeren RJ, Brüggen M, Vazza F, Bonafede A, Intema HT (2014) A new double radio relic in PSZ1 G096.89+24.17 and a radio relic mass-luminosity relation. MNRAS 444:3130-3138, DOI 10.1093/mnras/stu1658, 1408.2677

de Plaa J, Werner N, Bykov AM, Kaastra JS, Méndez M, Vink J, Bleeker JAM, Bonamente M, Peterson JR (2006) Chemical evolution in Sérsic 159-03 observed with XMM-Newton. A \& A 452:397-412, DOI 10.1051/0004-6361:20053864, astro-ph/0602582

Dolag K, Enßlin TA (2000) Radio halos of galaxy clusters from hadronic secondary electron injection in realistic magnetic field configurations. A \& A 362:151-157, arXiv:astro-ph/0008333

Donnert J, Brunetti G (2014) An efficient Fokker-Planck solver and its application to stochastic particle acceleration in galaxy clusters. MNRAS 443:3564-3577, DOI 
10.1093/mnras/stu1417, 1407.2735

Donnert J, Dolag K, Cassano R, Brunetti G (2010) Radio haloes from simulations and hadronic models - II. The scaling relations of radio haloes. MNRAS 407:15651580, DOI 10.1111/j.1365-2966.2010.17065.x, 1003.0336

Donnert J, Dolag K, Brunetti G, Cassano R (2013) Rise and fall of radio haloes in simulated merging galaxy clusters. MNRAS 429:3564-3569, DOI 10.1093/mnras/ sts628, 1211.3337

Enßlin T, Pfrommer C, Miniati F, Subramanian K (2011) Cosmic ray transport in galaxy clusters: implications for radio halos, gamma-ray signatures, and cool core heating. A \& A 527:A99, DOI 10.1051/0004-6361/201015652, 1008.4717

Enßlin TA, Pfrommer C, Springel V, Jubelgas M (2007) Cosmic ray physics in calculations of cosmological structure formation. A \& A 473:41-57, DOI 10.1051/ 0004-6361:20065294, arXiv:astro-ph/0603484

Ettori S, Fabian AC (2006) Effects of sedimented helium on the X-ray properties of galaxy clusters. MNRAS 369:L42-L46, DOI 10.1111/j.1745-3933.2006.00170.x, astro-ph/0603383

Fabian AC, Pringle JE (1977) On the spatial distribution of heavy elements in X-ray emitting clusters of galaxies. MNRAS 181:5P-7P, DOI 10.1093/mnras/181.1.5P

Feretti L, Giovannini G, Govoni F, Murgia M (2012) Clusters of galaxies: observational properties of the diffuse radio emission. The Astronomy and Astrophysics Review 20:54, DOI 10.1007/s00159-012-0054-z, 1205.1919

Ferrari C, Govoni F, Schindler S, Bykov AM, Rephaeli Y (2008) Observations of Extended Radio Emission in Clusters. Space Science Reviews 134:93-118, DOI 10.1007/s11214-008-9311-x, 0801.0985

Fisk LA (2015) 50 years of research on particle acceleration in the heliosphere. In: Journal of Physics Conference Series, Journal of Physics Conference Series, vol 642, p 012009, DOI 10.1088/1742-6596/642/1/012009

Fisk LA, Gloeckler G (2006) The Common Spectrum for Accelerated Ions in the Quiet-Time Solar Wind. ApJL 640:L79-L82, DOI 10.1086/503293

Fisk LA, Gloeckler G (2014) The case for a common spectrum of particles accelerated in the heliosphere: Observations and theory. Journal of Geophysical Research (Space Physics) 119:8733-8749, DOI 10.1002/2014JA020426

Fisk LA, Gloeckler G (2017) The pump acceleration mechanism. In: Journal of Physics Conference Series, Journal of Physics Conference Series, vol 900, p 012006, DOI 10.1088/1742-6596/900/1/012006

Gargaté L, Bingham R, Fonseca RA, Silva LO (2007) dHybrid: A massively parallel code for hybrid simulations of space plasmas. Computer Physics Communications 176:419-425, DOI 10.1016/j.cpc.2006.11.013, physics/0611174

Giacalone J, Burgess D, Schwartz SJ, Ellison DC, Bennett L (1997) Injection and acceleration of thermal protons at quasi-parallel shocks: A hybrid simulation parameter survey. Journal of Geophysical Research 1102:19789-19804, DOI 10.1029/97JA01529

Gilfanov MR, Syunyaev RA (1984) Intracluster Gravitational Separation of Deuterium and Helium in Rich Galaxy Clusters. Soviet Astronomy Letters 10:137-140

Grenier IA, Black JH, Strong AW (2015) The Nine Lives of Cosmic Rays in Galaxies. ARAA 53:199-246, DOI 10.1146/annurev-astro-082214-122457 
Griffin RD, Dai X, Kochanek CS (2014) New Limits on Gamma-Ray Emission from Galaxy Clusters. ApJL 795:L21, DOI 10.1088/2041-8205/795/1/L21, 1405.7047

Guo X, Sironi L, Narayan R (2014a) Non-thermal Electron Acceleration in Low Mach Number Collisionless Shocks. I. Particle Energy Spectra and Acceleration Mechanism. ApJ 794:153, DOI 10.1088/0004-637X/794/2/153, 1406.5190

Guo X, Sironi L, Narayan R (2014b) Non-thermal Electron Acceleration in Low Mach Number Collisionless Shocks. II. Firehose-mediated Fermi Acceleration and its Dependence on Pre-shock Conditions. ApJ 797:47, DOI 10.1088/0004-637X/ 797/1/47, 1409.7393

Guo X, Sironi L, Narayan R (2014c) Non-Thermal Electron Acceleration in Low Mach Number Collisionless Shocks. II. Firehose-Mediated Fermi Acceleration and its Dependence on Pre-Shock Conditions. ArXiv e-prints 1409.7393

Guo X, Sironi L, Narayan R (2017) Electron Heating in Low-Mach-number Perpendicular Shocks. I. Heating Mechanism. ApJ 851:134, DOI 10.3847/1538-4357/ aa9b82, 1710.07648

Guo X, Sironi L, Narayan R (2018) Electron Heating in Low Mach Number Perpendicular Shocks. II. Dependence on the Pre-shock Conditions. ApJ 858:95, DOI 10.3847/1538-4357/aab6ad, 1712.03239

Ha JH, Ryu D, Kang H, van Marle AJ (2018) Proton Acceleration in Weak Quasiparallel Intracluster Shocks: Injection and Early Acceleration. ApJ 864:105, DOI 10.3847/1538-4357/aad634, 1807.09403

Hoeft M, Brüggen M (2007) Radio signature of cosmological structure formation shocks. MNRAS 375:77-91, DOI 10.1111/j.1365-2966.2006.11111.x, arXiv: astro-ph/0609831

Hoeft M, Brüggen M, Yepes G, Gottlöber S, Schwope A (2008) Diffuse radio emission from clusters in the MareNostrum Universe simulation. MNRAS 391:15111526, DOI 10.1111/j.1365-2966.2008.13955.x, 0807.1266

Hong SE, Kang H, Ryu D (2015) Radio and X-Ray Shocks in Clusters of Galaxies. ApJ 812:49, DOI 10.1088/0004-637X/812/1/49, 1504.03102

Jacob S, Pakmor R, Simpson CM, Springel V, Pfrommer C (2018) The dependence of cosmic ray-driven galactic winds on halo mass. MNRAS 475:570-584, DOI 10.1093/mnras/stx3221, 1712.04947

Jokipii JR, Lee MA (2010) Compression Acceleration in Astrophysical Plasmas and the Production of $\mathrm{f}(\mathrm{v})$ vprop $\mathrm{v}^{-5}$ Spectra in the Heliosphere. ApJ 713:475-483, DOI 10.1088/0004-637X/713/1/475

Jubelgas M, Springel V, Enßlin T, Pfrommer C (2008) Cosmic ray feedback in hydrodynamical simulations of galaxy formation. A \& A 481:33-63, DOI 10.1051/ 0004-6361:20065295, arXiv:astro-ph/0603485

Kaastra JS, Bykov AM, Werner N (2009) Non-Maxwellian electron distributions in clusters of galaxies. A \& A 503:373-378, DOI 10.1051/0004-6361/200912492, 0905.4802

Kang H, Jones TW (2007) Self-similar evolution of cosmic-ray-modified quasiparallel plane shocks. Astroparticle Physics 28:232-246, DOI 10.1016/j. astropartphys.2007.05.007, 0705.3274

Kang H, Ryu D (2013) Diffusive Shock Acceleration at Cosmological Shock Waves. ApJ 764:95, DOI 10.1088/0004-637X/764/1/95, 1212.3246 
Kang H, Ryu D (2018) Effects of Alfvénic Drift on Diffusive Shock Acceleration at Weak Cluster Shocks. ApJ 856:33, DOI 10.3847/1538-4357/aab1f2, 1802.03189

Kang H, Ryu D, Cen R, Ostriker JP (2007) ApJ 669:729-740, DOI 10.1086/521717, arXiv:0704.1521

Kang H, Ryu D, Jones TW (2012a) Diffusive Shock Acceleration Simulations of Radio Relics. ApJ 756:97, DOI 10.1088/0004-637X/756/1/97, 1205.1895

Kang H, Ryu D, Jones TW (2012b) Diffusive Shock Acceleration Simulations of Radio Relics. ApJ 756:97, DOI 10.1088/0004-637X/756/1/97, 1205.1895

Kato TN, Takabe H (2010) Nonrelativistic Collisionless Shocks in Weakly Magnetized Electron-Ion Plasmas: Two-dimensional Particle-in-cell Simulation of Perpendicular Shock. ApJ 721:828-842, DOI 10.1088/0004-637X/721/1/828, 1008. 0265

Katsuta J, Uchiyama Y, Funk S (2017) Extended Gamma-Ray Emission from the G25.0+0.0 Region: A Star-forming Region Powered by the Newly Found OB Association? ApJ 839:129, DOI 10.3847/1538-4357/aa6aa3, 1704.06110

Keshet U, Waxman E, Loeb A (2004) Imprint of Intergalactic Shocks on the Radio Sky. ApJ 617:281-302, DOI 10.1086/424837, astro-ph/0402320

Komarov SV, Churazov EM, Kunz MW, Schekochihin AA (2016) Thermal conduction in a mirror-unstable plasma. MNRAS 460:467-477, DOI 10.1093/mnras/ stw963, 1603.00524

Kropotina JA, Bykov AM, Krassilchtchikov AM, Levenfish KP (2018) Maximus: a Hybrid Particle-in-Cell Code for Microscopic Modeling of Collisionless Plasmas. ArXiv e-prints 1806.05926

Kropotina YA, Bykov AM, Krasil'shchikov AM, Levenfish KP (2016) Relaxation of heavy ions in collisionless shock waves in cosmic plasma. Journal of Technical Physics 61:517-524, DOI 10.1134/S1063784216040149

Kunz MW, Schekochihin AA, Stone JM (2014) Firehose and Mirror Instabilities in a Collisionless Shearing Plasma. Physical Review Letters 112(20):205003, DOI 10.1103/PhysRevLett.112.205003, 1402.0010

Kushnir D, Waxman E (2009) Nonthermal emission from clusters of galaxies. Journal of Cosmology and Astroparticle Physics 8:002, DOI 10.1088/1475-7516/2009/08/ 002,0903.2271

Lazarian A, Brunetti G (2011) Turbulence, reconnection and cosmic rays in galaxy clusters . Memorie della Societá Astronomica Italiana 82:636, 1108.2268

Lingenfelter RE (2017) Cosmic rays from supernova remnants and superbubbles. Advances in Space Research

Lipatov AS (2002) The hybrid multiscale simulation technology: an introduction with application to astrophysical and laboratory plasmas

Marcowith A, Bret A, Bykov A, Dieckman ME, O'C Drury L, Lembège B, Lemoine M, Morlino G, Murphy G, Pelletier G, Plotnikov I, Reville B, Riquelme M, Sironi L, Stockem Novo A (2016) The microphysics of collisionless shock waves. Reports on Progress in Physics 79(4):046901, DOI 10.1088/0034-4885/79/4/046901, 1604.00318

Markevitch M, Vikhlinin A (2007) Shocks and cold fronts in galaxy clusters. Physics Reports 443:1-53, DOI 10.1016/j.physrep.2007.01.001, astro-ph/0701821

Markevitch M, Govoni F, Brunetti G, Jerius D (2005) Bow Shock and Radio Halo in 
the Merging Cluster A520. ApJ 627:733-738, DOI 10.1086/430695, astro-ph/ 0412451

Martin-Alvarez S, Planelles S, Quilis V (2017) On the interplay between cosmological shock waves and their environment. Astrophys. Space Sci. 362:91, DOI 10.1007/s10509-017-3066-3

Masters A, Sulaiman AH, Stawarz Ł, Reville B, Sergis N, Fujimoto M, Burgess D, Coates AJ, Dougherty MK (2017) An in situ Comparison of Electron Acceleration at Collisionless Shocks under Differing Upstream Magnetic Field Orientations. ApJ 843:147, DOI 10.3847/1538-4357/aa76ea, 1705.11096

Matsukiyo S, Matsumoto Y (2015) Electron Acceleration at a High Beta and Low Mach Number Rippled Shock. In: Journal of Physics Conference Series, Journal of Physics Conference Series, vol 642, p 012017, DOI 10.1088/1742-6596/642/1/ 012017

Matsumoto H (1977) Test particle study of nonlinear wave-particle interaction in the magnetosonic mode - Pure sinusoidal wave model. Physics of Fluids 20:20932103, DOI 10.1063/1.861839

Matthews AP (1994) Current Advance Method and Cyclic Leapfrog for 2D Multispecies Hybrid Plasma Simulations. Journal of Computational Physics 112:102 116, DOI 10.1006/jcph.1994.1084

Medvedev PS, Sazonov SY, Gilfanov MR (2017) Diffusion of elements in the interstellar medium in early-type galaxies. Astronomy Letters 43:285-303, DOI 10.1134/S1063773717050024, 1802.03217

Mernier F, de Plaa J, Kaastra JS, Zhang YY, Akamatsu H, Gu L, Kosec P, Mao J, Pinto C, Reiprich TH, Sanders JS, Simionescu A, Werner N (2017) Radial metal abundance profiles in the intra-cluster medium of cool-core galaxy clusters, groups, and ellipticals. A \& A 603:A80, DOI 10.1051/0004-6361/201630075, 1703.01183

Miley G (1980) The structure of extended extragalactic radio sources. ARAA 18:165-218, DOI 10.1146/annurev.aa.18.090180.001121

Miniati F (2003) Numerical modelling of gamma radiation from galaxy clusters. MNRAS 342:1009-1020, DOI 10.1046/j.1365-8711.2003.06647.x, arXiv: astro-ph/0303593

Miniati F, Ryu D, Kang H, Jones TW, Cen R, Ostriker JP (2000) ApJ 542:608-621, DOI 10.1086/317027, arXiv:astro-ph/0005444

Miniati F, Jones TW, Kang H, Ryu D (2001a) ApJ 562:233-253, DOI 10.1086/ 323434, arXiv: astro-ph/0108305

Miniati F, Jones TW, Kang H, Ryu D (2001b) Cosmic-Ray Electrons in Groups and Clusters of Galaxies: Primary and Secondary Populations from a Numerical Cosmological Simulation. ApJ 562:233-253, DOI 10.1086/323434, astro-ph/ 0108305

Mouhot C, Villani C (2010) Landau damping. Journal of Mathematical Physics 51(1):015204-015204, DOI 10.1063/1.3285283, 0905.2167

Nuza SE, Hoeft M, van Weeren RJ, Gottlöber S, Yepes G (2012) How many radio relics await discovery? MNRAS 420:2006-2019, DOI 10.1111/j.1365-2966.2011. 20118.x, 1111.1721

Nuza SE, Gelszinnis J, Hoeft M, Yepes G (2017) Can cluster merger shocks reproduce the luminosity and shape distribution of radio relics? MNRAS 470:240-263, 
DOI 10.1093/mnras/stx1109, 1704.06661

Oka M, Terasawa T, Seki Y, Fujimoto M, Kasaba Y, Kojima H, Shinohara I, Matsui H, Matsumoto H, Saito Y, Mukai T (2006) Whistler critical Mach number and electron acceleration at the bow shock: Geotail observation. Geophysical Research Letters 33:L24104, DOI 10.1029/2006GL028156

O'Neil T (1965) Collisionless Damping of Nonlinear Plasma Oscillations. Physics of Fluids 8:2255-2262, DOI 10.1063/1.1761193

Petrosian V, Bykov AM (2008) Particle Acceleration Mechanisms. Space Science Reviews 134:207-227, DOI 10.1007/s11214-008-9315-6, 0801.0923

Pfrommer C, Enßlin TA (2004) Journal of Korean Astronomical Society 37:455-460, arXiv: astro-ph/0412371

Pfrommer C, Springel V, Enßlin TA, Jubelgas M (2006) MNRAS 367:113-131, DOI 10.1111/j.1365-2966.2005.09953.x, arXiv: astro-ph/0603483

Pfrommer C, Enßlin TA, Springel V, Jubelgas M, Dolag K (2007) MNRAS 378:385408, DOI 10.1111/j.1365-2966.2007.11732.x, arXiv: astro-ph/0611037

Pfrommer C, Enßlin TA, Springel V (2008) Simulating cosmic rays in clusters of galaxies - II. A unified scheme for radio haloes and relics with predictions of the $\gamma$ ray emission. MNRAS 385:1211-1241, DOI 10.1111/j.1365-2966.2008.12956.x, 0707.1707

Pinzke A, Pfrommer C (2010) Simulating the $\gamma$-ray emission from galaxy clusters: a universal cosmic ray spectrum and spatial distribution. MNRAS 409:449-480, DOI 10.1111/j.1365-2966.2010.17328.x, 1001.5023

Pinzke A, Oh SP, Pfrommer C (2013) Giant radio relics in galaxy clusters: reacceleration of fossil relativistic electrons? MNRAS 435:1061-1082, DOI 10.1093/mnras/ stt1308, 1301.5644

Pinzke A, Oh SP, Pfrommer C (2017) Turbulence and particle acceleration in giant radio haloes: the origin of seed electrons. MNRAS 465:4800-4816, DOI 10.1093/ mnras/stw3024, 1611.07533

Planelles S, Quilis V (2013) Cosmological shock waves: clues to the formation history of haloes. MNRAS 428:1643-1655, DOI 10.1093/mnras/sts142, 1210.1369

Planelles S, Mimica P, Quilis V, Cuesta-Martínez C (2018) Multiwavelength mock observations of the WHIM in a simulated galaxy cluster. MNRAS 476:4629-4648, DOI 10.1093/mnras/sty527, 1802.09458

Prokhorov DA, Churazov EM (2014) Counting gamma rays in the directions of galaxy clusters. A \& A 567:A93, DOI 10.1051/0004-6361/201322454, 1309. 0197

Quest KB (1988) Theory and simulation of collisionless parallel shocks. Journal of Geophysical Research 193:9649-9680, DOI 10.1029/JA093iA09p09649

Reimer O, Pohl M, Sreekumar P, Mattox JR (2003) EGRET Upper Limits on the High-Energy Gamma-Ray Emission of Galaxy Clusters. ApJ 588:155-164, DOI 10.1086/374046, arXiv: astro-ph/0301362

Ryu D, Kang H, Biermann PL (2003a) Dynamical Role of Cosmic Rays in Clusters of Galaxies. In: Bowyer S, Hwang CY (eds) Matter and Energy in Clusters of Galaxies, Astronomical Society of the Pacific Conference Series, vol 301, p 327

Ryu D, Kang H, Hallman E, Jones TW (2003b) Cosmological Shock Waves and Their Role in the Large-Scale Structure of the Universe. ApJ 593:599-610, DOI 
$10.1086 / 376723$, astro-ph/0305164

Salem M, Bryan GL, Corlies L (2016) Role of cosmic rays in the circumgalactic medium. MNRAS 456:582-601, DOI 10.1093/mnras/stv2641, 1511.05144

Schaal K, Springel V, Pakmor R, Pfrommer C, Nelson D, Vogelsberger M, Genel S, Pillepich A, Sijacki D, Hernquist L (2016) Shock finding on a moving-mesh II. Hydrodynamic shocks in the Illustris universe. MNRAS 461:4441-4465, DOI 10.1093/mnras/stw1587, 1604.07401

Schekochihin AA, Cowley SC, Kulsrud RM, Rosin MS, Heinemann T (2008) Nonlinear Growth of Firehose and Mirror Fluctuations in Astrophysical Plasmas. Physical Review Letters 100(8):081301, DOI 10.1103/PhysRevLett.100.081301, 0709.3828

Schlickeiser R (2002) Cosmic Ray Astrophysics, Springer

Shimada N, Terasawa T, Hoshino M, Naito T, Matsui H, Koi T, Maezawa K (1999) Diffusive Shock Acceleration of Electrons at an Interplanetary Shock Observed on 21 Feb 1994. Astrophys. Space Sci. 264:481-488, DOI 10.1023/A: 1002499513777

Skillman SW, Hallman EJ, O'Shea BW, Burns JO, Smith BD, Turk MJ (2011) Galaxy Cluster Radio Relics in Adaptive Mesh Refinement Cosmological Simulations: Relic Properties and Scaling Relationships. ApJ 735:96-+, DOI 10.1088/ 0004-637X/735/2/96, 1006.3559

Skillman SW, Xu H, Hallman EJ, O'Shea BW, Burns JO, Li H, Collins DC, Norman ML (2013) Cosmological Magnetohydrodynamic Simulations of Galaxy Cluster Radio Relics: Insights and Warnings for Observations. ApJ 765:21, DOI 10.1088/ 0004-637X/765/1/21, 1211.3122

Sunyaev RA, Zeldovich YB (1972) Formation of Clusters of Galaxies; Protocluster Fragmentation and Intergalactic Gas Heating. A \& A 20:189

The Fermi-LAT Collaboration, :, Ackermann M, Ajello M, Albert A, Allafort A, Atwood WB, Baldini L, Ballet J, Barbiellini G, Bastieri D, Bechtol K, Bellazzini R, Bloom ED, Bonamente E, Bottacini E, Brandt TJ, Bregeon J, Brigida M, Bruel P, Buehler R, Buson S, Caliandro GA, Cameron RA, Caraveo PA, Cavazzuti E, Chaves RCG, Chiang J, Chiaro G, Ciprini S, Claus R, Cohen-Tanugi J, Conrad J, D’Ammando F, de Angelis A, de Palma F, Dermer CD, Digel SW, Drell PS, DrlicaWagner A, Favuzzi C, Franckowiak A, Funk S, Fusco P, Gargano F, Gasparrini D, Germani S, Giglietto N, Giordano F, Giroletti M, Godfrey G, Gomez-Vargas GA, Grenier IA, Guiriec S, Gustafsson M, Hadasch D, Hayashida M, Hewitt J, Hughes RE, Jeltema TE, Jóhannesson G, Johnson AS, Kamae T, Kataoka J, Knödlseder J, Kuss M, Lande J, Larsson S, Latronico L, Llena Garde M, Longo F, Loparco F, Lovellette MN, Lubrano P, Mayer M, Mazziotta MN, McEnery JE, Michelson PF, Mitthumsiri W, Mizuno T, Monzani ME, Morselli A, Moskalenko IV, Murgia S, Nemmen R, Nuss E, Ohsugi T, Orienti M, Orlando E, Ormes JF, Perkins JS, Pesce-Rollins M, Piron F, Pivato G, Rainò S, Rando R, Razzano M, Razzaque S, Reimer A, Reimer O, Ruan J, Sánchez-Conde M, Schulz A, Sgrò C, Siskind EJ, Spandre G, Spinelli P, Storm E, Strong AW, Suson DJ, Takahashi H, Thayer JG, Thayer JB, Thompson DJ, Tibaldo L, Tinivella M, Torres DF, Troja E, Uchiyama Y, Usher TL, Vandenbroucke J, Vianello G, Vitale V, Winer BL, Wood KS, Zimmer S, Pfrommer C, Pinzke A (2013) Search for cosmic-ray induced \$ $\backslash$ gamma $\$$-ray 
emission in Galaxy Clusters. ArXiv e-prints 1308.5654

Toptygin IN (1985) Cosmic rays in interplanetary magnetic fields, Springer, the Netherlands

Trotta D, Burgess D (2019) Electron acceleration at quasi-perpendicular shocks in sub- and supercritical regimes: 2D and 3D simulations. MNRAS 482:1154, DOI $10.1093 / \mathrm{mnras} / \mathrm{sty} 2756,1808.00812$

van Weeren RJ, Röttgering HJA, Brüggen M, Hoeft M (2010) Particle Acceleration on Megaparsec Scales in a Merging Galaxy Cluster. Science 330:347, DOI 10. 1126/science.1194293, 1010.4306

Vazza F, Brüggen M (2014) Do radio relics challenge diffusive shock acceleration? MNRAS 437:2291-2296, DOI 10.1093/mnras/stt2042,1310.5707

Vazza F, Brunetti G, Gheller C (2009) Shock waves in Eulerian cosmological simulations: main properties and acceleration of cosmic rays. MNRAS 395:1333-1354, DOI 10.1111/j.1365-2966.2009.14691.x, 0808.0609

Vazza F, Brunetti G, Gheller C, Brunino R (2010) Massive and refined: A sample of large galaxy clusters simulated at high resolution. I: Thermal gas and properties of shock waves. New Astronomy 15:695-711, DOI 10.1016/j.newast.2010.05.003, 1003.5658

Vazza F, Dolag K, Ryu D, Brunetti G, Gheller C, Kang H, Pfrommer C (2011) A comparison of cosmological codes: properties of thermal gas and shock waves in large-scale structures. MNRAS 418:960-985, DOI 10.1111/j.1365-2966.2011. 19546.x, 1106.2159

Vazza F, Brüggen M, Gheller C, Brunetti G (2012a) Modelling injection and feedback of cosmic rays in grid-based cosmological simulations: effects on cluster outskirts. MNRAS p 2518, DOI 10.1111/j.1365-2966.2012.20562.x, 1201.3362

Vazza F, Brüggen M, van Weeren R, Bonafede A, Dolag K, Brunetti G (2012b) Why are central radio relics so rare? MNRAS 421:1868-1873, DOI 10.1111/j. 1365-2966.2011.20160.x, 1111.1720

Vazza F, Brüggen M, Gheller C (2013) Thermal and non-thermal traces of AGN feedback: results from cosmological AMR simulations. MNRAS 428:2366-2388, DOI $10.1093 / \mathrm{mnras} / \mathrm{sts} 213,1210.3541$

Vazza F, Gheller C, Brüggen M (2014) Simulations of cosmic rays in large-scale structures: numerical and physical effects. ArXiv e-prints 1401.4454

Vazza F, Eckert D, Brüggen M, Huber B (2015) Electron and proton acceleration efficiency by merger shocks in galaxy clusters. MNRAS 451:2198-2211, DOI $10.1093 / \mathrm{mnras} / \mathrm{stv} 1072,1505.02782$

Vazza F, Brüggen M, Wittor D, Gheller C, Eckert D, Stubbe M (2016) Constraining the efficiency of cosmic ray acceleration by cluster shocks. MNRAS 459:70-83, DOI 10.1093/mnras/stw584, 1603.02688

Vink J, Broersen S, Bykov A, Gabici S (2015) On the electron-ion temperature ratio established by collisionless shocks. A \& A 579:A13, DOI 10.1051/0004-6361/ 201424612, 1407.4499

Völk HJ, Atoyan AM (1999) Clusters of galaxies: magnetic fields and nonthermal emission. Astroparticle Physics 11:73-82, DOI 10.1016/S0927-6505(99)00029-8, arXiv: astro-ph/9812458

Völk HJ, Aharonian FA, Breitschwerdt D (1996) The Nonthermal Energy Content 
and Gamma-Ray Emission of Starburst Galaxies and Clusters of Galaxies. Space Science Reviews 75:279-297, DOI 10.1007/BF00195040

Walker SA, ZuHone J, Fabian A, Sanders J (2018) The split in the ancient cold front in the Perseus cluster. Nature Astronomy 2:292-296, DOI 10.1038/ s41550-018-0401-8, 1803.00898

Wiener J, Oh SP, Guo F (2013) Cosmic ray streaming in clusters of galaxies. MNRAS 434:2209-2228, DOI 10.1093/mnras/stt1163, 1303.4746

Wiener J, Pfrommer C, Oh SP (2017) Cosmic ray-driven galactic winds: streaming or diffusion? MNRAS 467:906-921, DOI 10.1093/mnras/stx127, 1608.02585

Wiener J, Zweibel EG, Oh SP (2018) High $\beta$ effects on cosmic ray streaming in galaxy clusters. MNRAS 473:3095-3103, DOI 10.1093/mnras/stx2603, 1706 . 08525

Winske D, Omidi N (1996) A nonspecialist's guide to kinetic simulations of space plasmas. Journal of Geophysical Research 1101:17287-17304, DOI 10.1029/ 96JA00982

Wittor D, Vazza F, Brüggen M (2016) Testing cosmic-ray acceleration with radio relics: a high-resolution study using MHD and tracers. ArXiv e-prints 1610.05305

Wittor D, Vazza F, Brüggen M (2017) Testing cosmic ray acceleration with radio relics: a high-resolution study using MHD and tracers. MNRAS 464:4448-4462, DOI 10.1093/mnras/stw2631, 1610.05305

Xu S, Lazarian A (2018) Resonance-broadened transit time damping of particles in MHD turbulence. ArXiv e-prints 1810.07726

Yang Z, Lu Q, Liu YD, Wang R (2018) Impact of Shock Front Rippling and Selfreformation on the Electron Dynamics at Low-Mach-number Shocks. ApJ 857:36, DOI 10.3847/1538-4357/aab714

Zandanel F, Ando S (2014) Constraints on diffuse gamma-ray emission from structure formation processes in the Coma cluster. MNRAS 440:663-671, DOI $10.1093 / \mathrm{mnras} / \mathrm{stu} 324,1312.1493$

Zandanel F, Pfrommer C, Prada F (2014) On the physics of radio haloes in galaxy clusters: scaling relations and luminosity functions. MNRAS 438:124-144, DOI $10.1093 / \mathrm{mnras} / \mathrm{stt} 2250,1311.4795$

Zandanel F, Tamborra I, Gabici S, Ando S (2015) High-energy gamma-ray and neutrino backgrounds from clusters of galaxies and radio constraints. A \& A 578:A32, DOI 10.1051/0004-6361/201425249, 1410.8697

Zhang M (2010) Acceleration of suprathermal particles by compressional plasma wave trains in the solar wind. Journal of Geophysical Research (Space Physics) 115:A12102, DOI 10.1029/2010JA015723

Zimbardo G, Perri S (2018) Understanding the radio spectral indices of galaxy cluster relics by superdiffusive shock acceleration. MNRAS 478:4922-4930, DOI 10.1093/mnras/sty 1438

ZuHone JA, Kunz MW, Markevitch M, Stone JM, Biffi V (2015) The Effect of Anisotropic Viscosity on Cold Fronts in Galaxy Clusters. ApJ 798:90, DOI 10.1088/0004-637X/798/2/90, 1406.4031 